\title{
Active Hybrid Control Algorithm with Sky-Hook Damping and Lead-Lag Phase Compensation for Multi-DOFs Ultra-Low Frequency Active Vibration Isolation System
}

\author{
Min Wang, Xiaoqing Li, and Xuedong Chen \\ The State Key Laboratory of Digital Manufacturing Equipment and Technology, Huazhong University of Science and Technology, \\ Wuhan, Hubei Province, China
}

Correspondence should be addressed to Xuedong Chen; chenxd@hust.edu.cn

Received 7 September 2016; Revised 17 November 2016; Accepted 25 December 2016; Published 17 January 2017

Academic Editor: Marcello Vanali

Copyright (c) 2017 Min Wang et al. This is an open access article distributed under the Creative Commons Attribution License, which permits unrestricted use, distribution, and reproduction in any medium, provided the original work is properly cited.

With the improvement of performance in the ultra-precision manufacturing engineering, the requirements for vibration isolation have become more stringent. In order to obtain a wider effective bandwidth and a higher performance of a multi-DOFs active vibration isolation system (AVIS), active hybrid control (AHC) technology is applied in this paper. AHC technology comprises a feedback active control (FBAC) technology and a feedforward active control (FFAC) technology. Absolute velocity feedback is employed to establish a sky-hook damping technology in FBAC technology. Velocity feedforward of base platform is adopted to build a lead-lag phase compensation (LLPC) technology in FFAC technology. Further, a coordinate vector conversion from unit level to system level is mentioned to describe the dynamic characteristic of the six DOFs AVIS applied in the ultra-precision field. And with the assistance of the transformed coordinate vector, the dynamic model of system level is built. Based on the establishment of the dynamic model and the research of AHC, an experimental platform which constitutes three vibration isolators and a real-time active control system is set up. The experimental results indicate that the amplitude of the resonant peak is further reduced significantly, compared to the general feedback control. And simultaneously active effective bandwidth is extended. AHC technology with sky-hook damping algorithm and LLPC control algorithm is verified to be more effective.

\section{Introduction}

Vibration isolation device is used to realize high isolation performance of multi-DOFs and broad frequency band in precision equipment, such as wafer stepper lithography machines, atomic force microscopes (AFM), space telescopes and interferometers, and laser communication system [1]. Passive vibration isolation is appropriate for many applications, which generally consists of one or several stages of mass-spring-damper systems introduced in the propagation path. Although a passive isolation system offers a simple and reliable means of protecting precision equipment from a vibration environment, it has performance limitations since its controllable frequency range is limited [2]. Simultaneously, with the continuous improvement of the ultra-precision machining and manufacturing equipment, the performance requirement for low frequency and ultra-low frequency vibration isolation is more stringent. At the same time, with the rapid development of smart sensors, smart actuators, and microprocessors, active vibration isolation is becoming more and more attractive.

Active vibration isolation has greatly improved the performance of the vibration isolation but it requires a sensoractuator pair and a corresponding active control system, which requires the active vibration isolation mechanism to have a simple structure, an efficient algorithm [3-5]. Therefore, it is more necessary to find an effective active control strategy for active control technology. According to the control structure, active control strategy can be classified into feedback control and feedforward control. Depending on different actual vibration isolation applications, different vibration control strategies are employed for different scenarios. Feedback control may not achieve sufficiently highspeed responses because of delays intrinsically contained in its strategy. To overcome the shortcoming, if we try a high-gain feedback for position control, the control system 
will become unstable [6]. Active hybrid control technology is introduced to obtain a wider effective frequency band and a higher performance. Two of the most commonly used series compensation strategies are PID (proportionalintegral-derivative) control and phase lead-lag compensation [7].

Absolute velocity feedback [8] control creates "sky-hook" damping to reduce the resonance peak effectively, but it cannot suppress the vibration except near the resonance frequencies [4]. And once the feedback gain coefficient is excessive, the system tends to be unstable. At this time, velocity feedforward of base platform appears in a significant effect $[6,9]$ against the forecast of the environment vibration or the direct disturbance.

With the assistance of LLPC $[7,10,11]$ control, it can be based on the feedback control to further expand the effective bandwidth of the active vibration isolation. The performance of the AVIS can be effectively improved. Once a signal which has a strong correlation with the disturbance input signal cannot be obtained by the controller, the feedforward controller not only can eliminate the influence of direct disturbance or base disturbance, but also may exert the counterforce and even cause the instability of the system.

In this paper, some various well-known algorithms in other or related fields are combined and applied in the different loops of active control, which contain the velocity loop (feedback loop and feedforward loop) and position loop. Compared with the fact that active control is performed with one of the algorithms, AHC can achieve a better performance on vibration isolation. At the same time, with the PNSP structure employed in this paper, it can produce the effect that the natural frequency is reduced to ultra-low frequency $1.15 \mathrm{~Hz} \sim 1.20 \mathrm{~Hz}$ (in [12] it is $3 \mathrm{~Hz}$ and in [13] it is $4.96 \mathrm{~Hz}$ ), and the amplitude of the resonance peak and other bandwidths is greatly decreased by $23.58 \mathrm{~dB} \sim 29.17 \mathrm{~dB}$ (in [14] it is $10 \mathrm{~dB} \sim$ $20 \mathrm{~dB}$ ). Meanwhile, the vibration attenuation rate in $110 \mathrm{~Hz}$ is $98 \%$ (in [15] it is 54.04\%). And the active effective bandwidth is extended to the midfrequency region of $0.23 \mathrm{~Hz} \sim 45.74 \mathrm{~Hz}$ (in [12] it is $0.3 \mathrm{~Hz} 30 \mathrm{~Hz}$ ). Simultaneously, AHC algorithm can ensure the high performance of additional frequency range is unchanged. At the same time, it can obtain significant benefits in different DOFs through the independent adjustment of the parameters of each DOF with the assistance of MIMO to SISO, which play a more obvious role in the reality.

In this paper, the layout of the ultra-precision AVIS is introduced firstly in Section 2. Taking this into account, coordinate vector conversion from unit level to system level is mentioned. Simultaneously, a system level dynamic model is built in Section 3. Further, active hybrid control technology which comprises a FFAC technology and a FBAC technology is introduced in Section 4. In Section 5, an experimental platform of six DOFs active AVIS which consists of three isolators is set up in super-clean lab. Experimental results from the frequency domain and time domain are also discussed, respectively, in this section. Conclusions are summarised in Section 6.

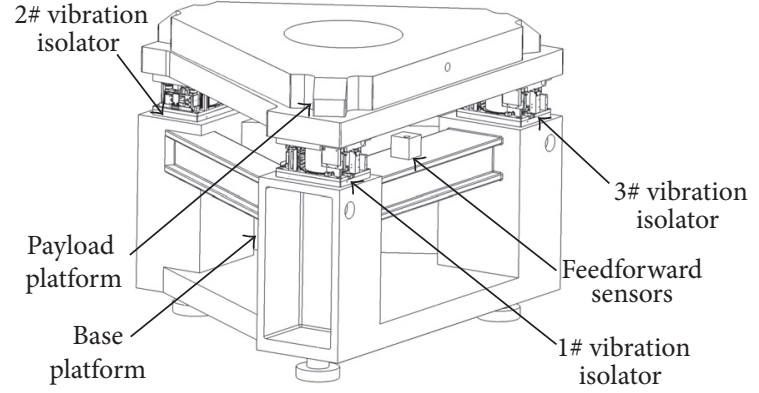

FIGURE 1: The prototype of the ultra-precision AVIS.

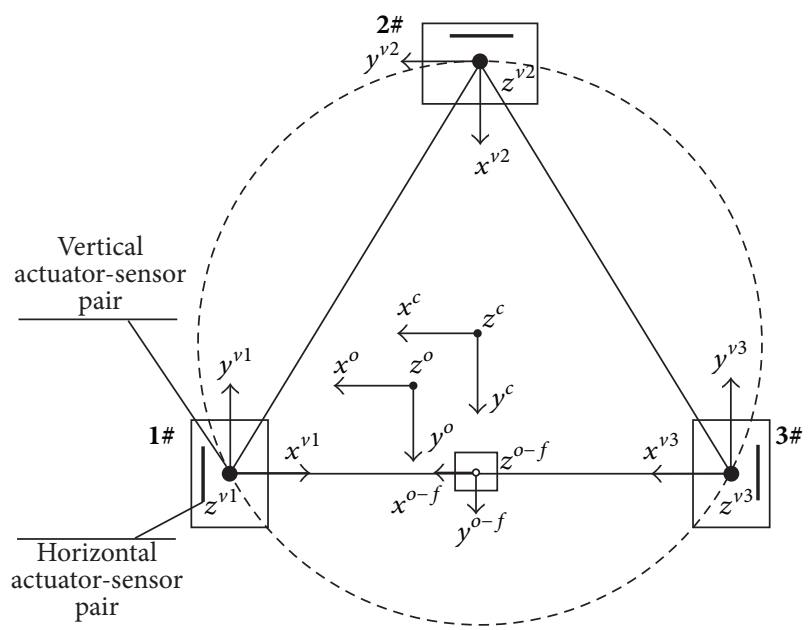

FIgURE 2: The layout of the AVIS coordinate system.

\section{The Profile of the AVIS}

2.1. The Layout of AVIS. AVIS is employed to isolate the vibration disturbance from the ground and ensure the working environment of the motion equipment or measuring equipment mounted on the payload platform. The prototype of the ultra-precision AVIS is shown in Figure 1.

AVIS consists of a payload platform used as the working platform, a base platform as the foundation frame, three identical vibration isolators as the main component of active vibration isolation, and a feedforward sensors device which can supply the input signal of FFAC. Three identical vibration isolators which are as equilateral triangle arrangement are installed on the three corners of the base platform. The feedforward sensors device is mounted on the base platform to collect the vibration signal of ground. The ultra-precision motion equipment or ultra-precision measuring equipment can be fitted on the payload platform with high stiffness.

Figure 2 elaborates the layout of the AVIS coordinate system. In Figure 2, the definition of the coordinate system is as follows: the global inertial coordinate system $\sum O$ is established and called the logical axis coordinate, the local coordinate system of vibration isolator $\sum V$ is described as the physical axis coordinate, and $\sum C$ is the centroid inertial coordinate system. 


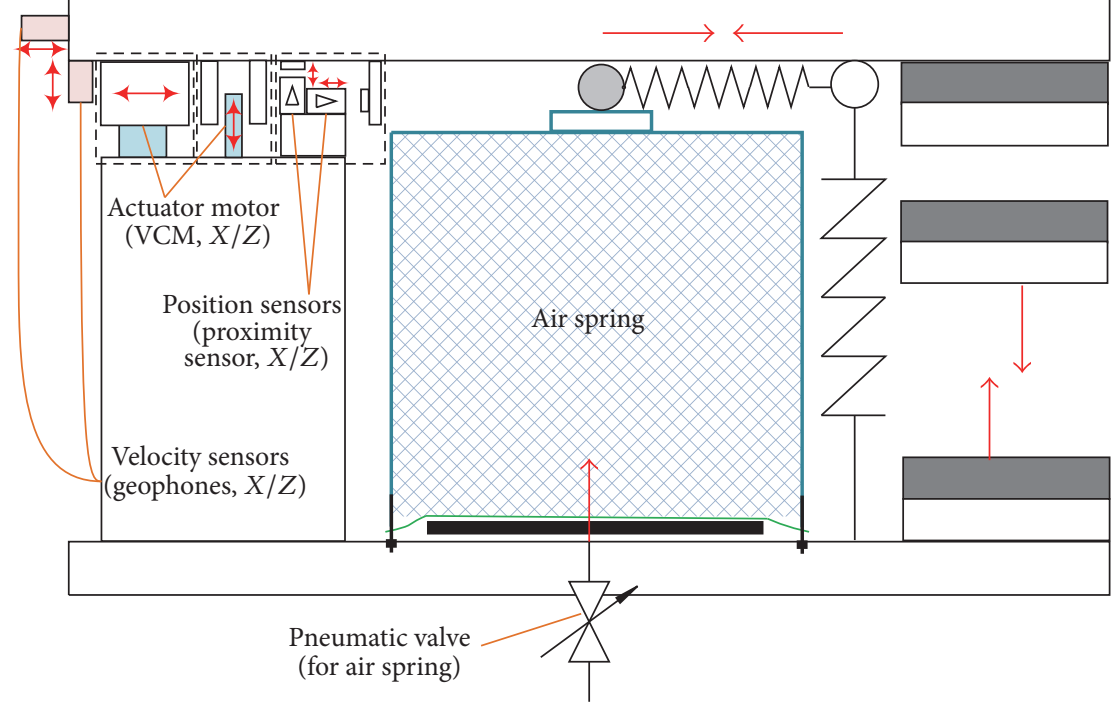

FIGURE 3: Schematic drawing of vibration isolator.

Based on this, the layout of the vibration isolator is as follows. The installation of $1 \#$ isolator and $3 \#$ isolator should be parallel to the $y$-axis of the global inertial coordinate system. On the contrary, 2\# isolator is parallel to the $x$-axis and mounted on the intersection which is formed by the equivalent diameter circle and the perpendicular bisector of $1 \#$ and 3\#. The layout is served for the formation of six DOFs of AVIS.

2.2. The Ultra-Low Frequency Vibration Isolator. The frequency range over which a linear passive vibration isolator is effective is often limited by the amount stiffness required to support a static load. This can be improved upon by incorporating a negative stiffness element to form a high-staticlow-dynamic stiffness mounts, which can be approximated to achieve the quasi-zero stiffness $[16,17]$ system. Figure 3 shows the schematic drawing of vibration isolator used throughout this paper. The vibration isolator with a structure of positive and negative stiffness in parallel (PNSP) in Figure 3 is adopted for the AVIS.

The ultra-precision vibration isolator contains a proximity sensor, a geophone, and a voice coil motor in vertical and horizontal direction, respectively. In addition, the ultraprecision vibration isolator also contains a pneumatic valve in the vertical direction. The signal collected via a position sensor is processed by the active control algorithm of the position loop controller. Active control signal is served for the input of pneumatic valve, so as to implement precision positioning control. The signal collected by a geophone is used for the active control algorithm of the velocity loop controller. Active control signals are served for the input of voice coil motors, so as to implement precision vibration isolation control.

According to the structure sketch of Figure 3, the schematic diagram of stiffness in parallel is illustrated in
Figure 4 separately from the horizontal and vertical direction. In vertical direction, a novel structure which combines a PS air spring with a NS magnetic spring in parallel is mentioned. The PS air spring can support the mass of payload platform, and the NS magnetic spring is responsible for realizing ultralow frequency vibration isolation. The stiffness of the system can be changed by adjusting the position of the middle magnet of NS magnetic spring.

In horizontal direction, a novel structure which combines a PS flat spring with a NS inverted pendulum in parallel is utilized to attain ultra-low frequency vibration isolation. The NS inverted pendulum is realized through the reverse installing of the air spring. When the payload is very large (about $1000 \mathrm{~kg}$ ), the cross-sectional area of the air spring is relatively small, which can be equivalent to simply supported beam structure. When one end of the simply supported beam is installed on the base platform and can be rotated, the other end is connected with the payload platform and also can be rotated. A pendulum structure that adopts inverted installation is formed.

At the same time, in order to eliminate the influence of the force from the other directions, a flexible joint with lower bending stiffness in the DOFs not required is adopted to serve for horizontal and vertical motion decoupling. A two DOFs' ultra-low frequency vibration isolator is set up thereby.

After PNSP, the stiffness of horizontal and vertical direction can be expressed as

$$
\begin{aligned}
& k_{\eta}^{V}=K^{V}=K_{\mathrm{po}}^{V}+K_{\mathrm{ne}}^{V}=K_{\mathrm{as}}+K_{\mathrm{ms}}, \\
& k_{\eta}^{H}=K^{H}=K_{\mathrm{po}}^{H}+K_{\mathrm{ne}}^{H}=K_{\mathrm{fs}}+K_{\mathrm{ip}},
\end{aligned}
$$

where $k_{\eta}^{V}=K^{V}$ is the system stiffness of vertical direction, $K_{\text {po }}^{V}$ is the positive stiffness of vertical direction, $K_{\text {ne }}^{V}$ is the negative stiffness of vertical direction, $K_{\mathrm{as}}$ is the stiffness of 


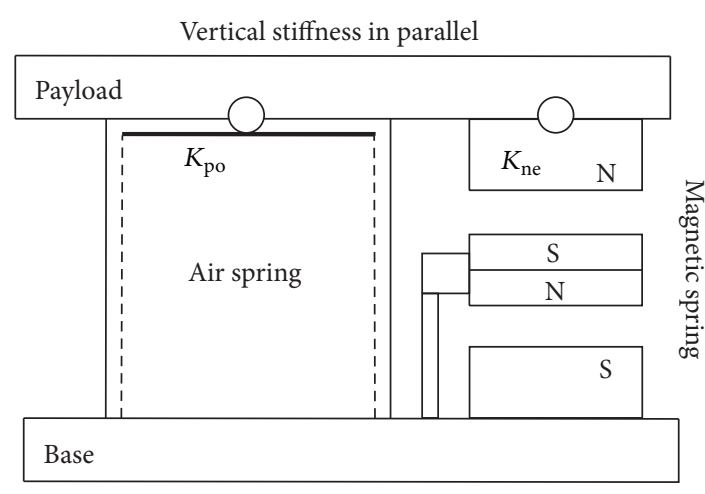

(a)

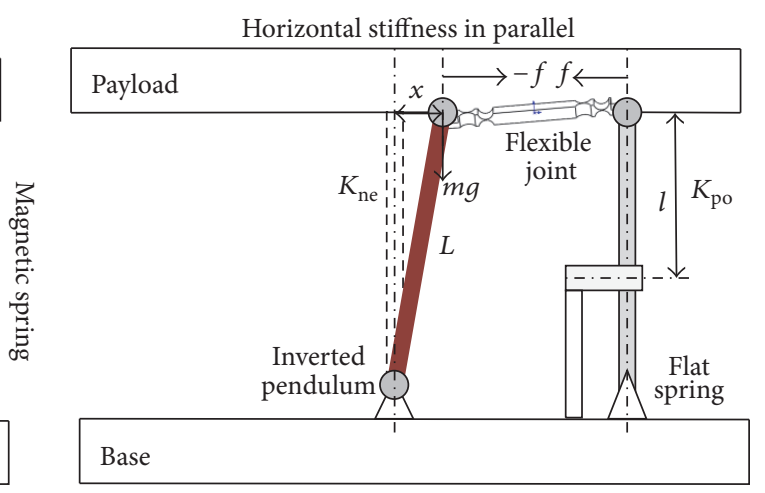

(b)

FIgURE 4: The schematic diagram of stiffness in parallel: (a) vertical direction and (b) horizontal direction.

air spring, $K_{\mathrm{ms}}$ is the stiffness of magnetic spring, $k_{\eta}^{H}=K^{H}$ is the system stiffness of horizontal direction, $K_{\text {po }}^{H}$ is the positive stiffness of horizontal direction, $K_{\mathrm{ne}}^{H}$ is the negative stiffness of horizontal direction, $K_{\mathrm{fs}}$ is the stiffness of flat spring, and $K_{\text {ip }}$ is the stiffness of inverted pendulum.

\section{Modeling of the AVIS}

3.1. The Coordinate Vector Conversion of AVIS. A six DOFs' vector $\mathbf{x}$ which is composed of translational displacement $\mathbf{r}$ and rotational displacement $\boldsymbol{\theta}$ is defined to describe the motion of the payload platform in the global inertial coordinate system $\sum O$ also called the logical axis coordinate. Figure 5 describes the geometrical relationship between the logic axis and the physical axis of the AVIS, where $C$ is the centroid of payload platform.

According to the block diagram of Figure 5, real physical axis signals measured by six feedback sensors are converted to the logic axis coordinate signals of the centroid by the transform matrix $\mathbf{T}_{S}$. After the successful meeting of the logic feedback signals and the feedforward sensor signals, the composite signals are sent into the AHC controller to perform separately with different control algorithms. The calculated signals are still the logic axis signals of the centroid. Therefore it is necessary to switch these signals to the physical axis control signals which can be used by the various vibration isolators through another transform matrix $\mathbf{T}_{A}$. The physical axis control signals are employed to drive different VCMs to achieve the entire closed loop active vibration isolation control. The direction of the sensor-actuator of Figure 5 is defined in Table 1.

The logical axis vector $\mathbf{x}$ for describing the rigid body motion of the AVIS can be expressed as

$$
\mathbf{x}=\left(\begin{array}{ll}
\mathbf{r} & \theta
\end{array}\right)=\left(\begin{array}{llllll}
x & y & \gamma & z & \beta & \alpha
\end{array}\right)^{T},
$$

where $x, y$, and $z$ are the translational displacements of the centroid in the Cartesian coordinate. $\alpha, \beta$, and $\gamma$ are the rotational displacements around the $x-, y$-, and $z$-axis in the Cardan angle coordinate.

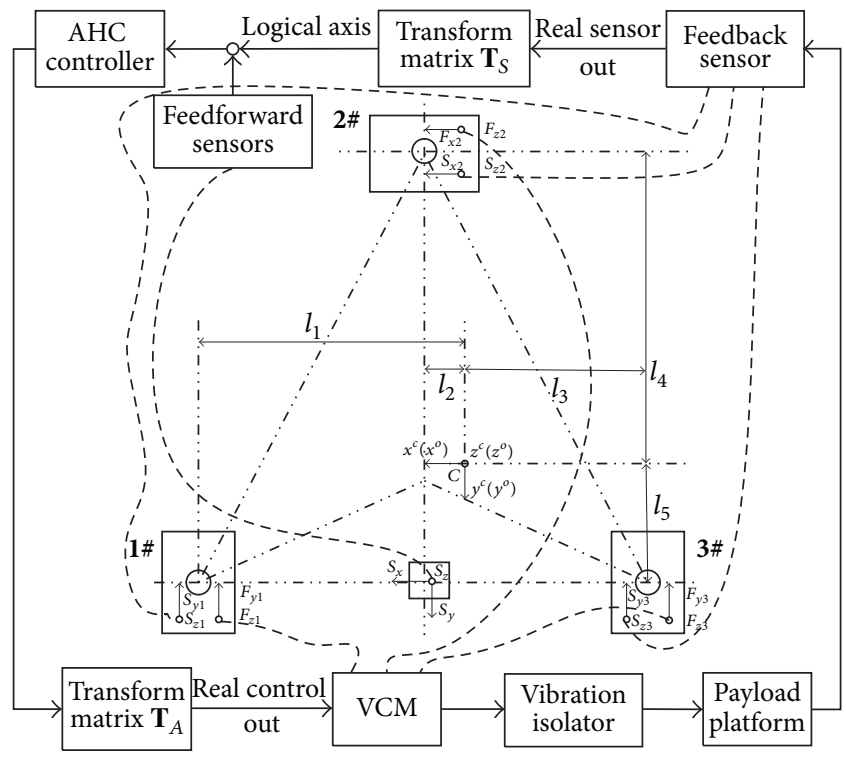

FIGURE 5: The layout of the actuator-sensor pairs.

TABLE 1: Sensor/actuator direction definition.

\begin{tabular}{lc}
\hline Sensor/actuator number & Direction \\
\hline$S_{y 1} / F_{y 1}$ & $-Y$ \\
$S_{x 2} / F_{x 2}$ & $+X$ \\
$S_{y 3} / F_{y 3}$ & $-Y$ \\
$S_{z 1} / F_{z 1}$ & $+Z$ \\
$S_{z 2} / F_{z 2}$ & $+Z$ \\
$S_{z 3} / F_{z 3}$ & $+Z$ \\
\hline
\end{tabular}

The physical axis coordinate vector can be expressed as

$$
\mathbf{q}=\left(\begin{array}{llllll}
s_{y 1} & s_{x 2} & s_{y 3} & s_{z 1} & s_{z 2} & \left.s_{z 3}\right)^{T},
\end{array}\right.
$$

where $s_{y 1}, s_{x 2}$, and $s_{y 3}$ are the signals measured by horizontal feedback sensors and $s_{z 1}, s_{z 2}$, and $s_{z 3}$ are the signals from vertical feedback sensors. 
When the vibration amplitude of the payload is relatively small, there is the following transformation:

$$
\mathbf{x}=\mathbf{T}_{S} \mathbf{q}
$$

where $\mathbf{T}_{S}$ is the sensor transform matrix which describes the conversion relationship from the physical axis to the logical axis

$$
\mathbf{T}_{S}=\left(\begin{array}{cccccc}
\frac{l_{4}}{l_{1}+l_{3}} & 1 & -\frac{l_{4}}{l_{1}+l_{3}} & 0 & 0 & 0 \\
-\frac{l_{3}}{l_{1}+l_{3}} & 0 & -\frac{l_{1}}{l_{1}+l_{3}} & 0 & 0 & 0 \\
-\frac{1}{l_{1}+l_{3}} & 0 & \frac{1}{l_{1}+l_{3}} & 0 & 0 & 0 \\
0 & 0 & 0 & \frac{l_{3} l_{4}-l_{2} l_{5}}{\left(l_{1}+l_{3}\right)\left(l_{4}+l_{5}\right)} & \frac{l_{5}}{l_{4}+l_{5}} & \frac{l_{1} l_{4}-l_{2} l_{5}}{\left(l_{1}+l_{3}\right)\left(l_{4}+l_{5}\right)} \\
0 & 0 & 0 & -\frac{1}{l_{1}+l_{3}} & 0 & \frac{1}{l_{1}+l_{3}} \\
0 & 0 & 0 & \frac{l_{2}+l_{3}}{\left(l_{1}+l_{3}\right)\left(l_{4}+l_{5}\right)} & -\frac{1}{l_{4}+l_{5}} & \frac{l_{1}+l_{2}}{\left(l_{1}+l_{3}\right)\left(l_{4}+l_{5}\right)}
\end{array}\right) .
$$

Similarly, when the vibration amplitude is very small, there is the following transformation about the force coordinate vector:

$$
\mathbf{F}_{p}=\mathbf{T}_{A} \mathbf{F}_{l}=\mathbf{T}_{A}\left(\begin{array}{ll}
\mathbf{F}_{c} & \mathbf{M}_{c}
\end{array}\right),
$$

where $\mathbf{F}_{p}$ is the physical coordinate vector of Lorentz motors, $\mathbf{F}_{l}$ is the logical axis force vector, $\mathbf{F}_{c}$ is the force vector at the centroid, $\mathbf{M}_{c}$ is the moment vector around the centroid, and $\mathbf{T}_{A}$ is the actuator conversion matrix which describes the conversion relationship from the physical axis force vector to the logical axis force vector

$$
\mathbf{T}_{A}=\left(\begin{array}{cccccc}
\frac{l_{4}}{l_{1}+l_{3}} & -\frac{l_{3}}{l_{1}+l_{3}} & -\frac{1}{l_{1}+l_{3}} & 0 & 0 & 0 \\
1 & 0 & 0 & 0 & 0 & 0 \\
-\frac{l_{4}}{l_{1}+l_{3}} & -\frac{l_{1}}{l_{1}+l_{3}} & \frac{1}{l_{1}+l_{3}} & 0 & 0 & 0 \\
0 & 0 & 0 & \frac{l_{3} l_{4}-l_{2} l_{5}}{\left(l_{1}+l_{3}\right)\left(l_{4}+l_{5}\right)} & -\frac{1}{l_{1}+l_{3}} & \frac{l_{2}+l_{3}}{\left(l_{1}+l_{3}\right)\left(l_{4}+l_{5}\right)} \\
0 & 0 & 0 & \frac{l_{5}}{l_{4}+l_{5}} & 0 & -\frac{1}{l_{4}+l_{5}} \\
0 & 0 & 0 & \frac{l_{1} l_{4}-l_{2} l_{5}}{\left(l_{1}+l_{3}\right)\left(l_{4}+l_{5}\right)} & \frac{1}{l_{1}+l_{3}} & \frac{l_{1}+l_{2}}{\left(l_{1}+l_{3}\right)\left(l_{4}+l_{5}\right)}
\end{array}\right) .
$$

3.2. The Simplified Dynamic Model of AVIS. The dynamic characteristic of the AVIS is mainly determined by the passive vibration isolation unit. Therefore, each isolator can be simplified as a combination of the elastic element and damping element in three directions $X, Y$, and $Z$. The simplified dynamic model of the system is shown in Figure 6, where $K_{D i}(D=x, y, z ; i=1,2,3)$ is the stiffness of the $i$ th isolator in the $D$ direction and $C_{D i}(D=x, y, z ; i=1,2,3)$ is the damping of the $i$ th isolator in the $D$ direction.

The logical axis displacement vector $\mathbf{x}$ and force vector $\mathbf{F}_{l}$ are defined as above. According to Newton-Euler equation, the dynamic equation of the AVIS can be written as

$$
\begin{aligned}
M \ddot{\mathbf{r}} & =\sum \mathbf{F}=\mathbf{F}_{c}+\mathbf{F}_{\text {iner }}, \\
\mathbf{J} \ddot{\boldsymbol{\theta}} & =\sum \mathbf{M}=\mathbf{M}_{c}+\mathbf{M}_{\text {iner }},
\end{aligned}
$$

where $M$ is the mass of the payload, $\mathbf{J}$ is the moment of inertia vector, $\mathbf{F}_{\text {iner }}$ is the spring force and the damping force from the isolator, and $\mathbf{M}_{\text {iner }}$ is the moment of the spring force and damping force converted to the centroid of the payload platform.

According to the definition of the spring force and the linear viscous damping force, the resultant force $\mathbf{F}_{\text {iner }}$ can be obtained from [18]

$$
\mathbf{F}_{\text {iner }}=-\sum_{i=1}^{3}\left(\mathbf{K}_{i} \mathbf{X}_{i}+\mathbf{C}_{i} \dot{\mathbf{X}}_{i}\right),
$$

where $i(i=1,2,3)$ is the number of vibration isolators, $\mathbf{K}_{i}=\operatorname{diag}\left(k_{x i}, k_{y i}, k_{z i}\right)$ is the stiffness matrix, and $\mathbf{C}_{i}=$ $\operatorname{diag}\left(c_{x i}, c_{y i}, c_{z i}\right)$ is the damping matrix of the three isolators. 


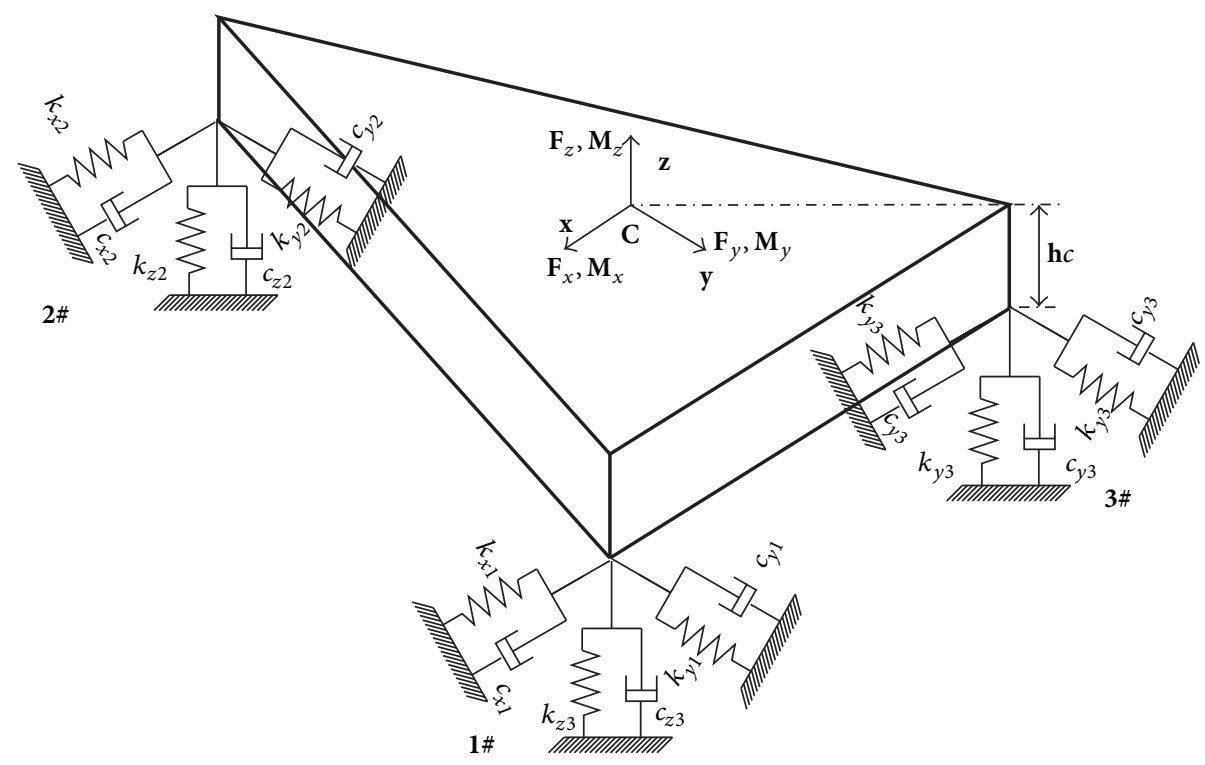

Figure 6: The simplified dynamic model of the system.

The resultant force moment around the centroid of the payload can be expressed as the cross product of the displacement vector and the force vector. The moment of the resultant force $\mathbf{M}_{\text {iner }}$ can be written from [18]

$$
\mathbf{M}_{\text {iner }}=\sum_{i=1}^{3}\left[\mathbf{p}_{i}^{C} \times\left(\mathbf{K}_{i} \mathbf{X}_{i}+\mathbf{C}_{i} \dot{\mathbf{X}}_{i}\right)\right],
$$

where $\mathbf{p}_{i}^{C}$ is the coordinates of the $i$ th isolator in the rigid body inertial coordinate system.

The dynamic equation describing the rigid body motion of the payload in AVIS can be deduced as

$$
\begin{aligned}
M \ddot{\mathbf{r}} & =\mathbf{F}_{c}+\sum_{i=1}^{3}\left[\mathbf{C}_{i}\left(\dot{\mathbf{r}}+\mathbf{R}^{C O}(\theta) \dot{\mathbf{p}}_{i}^{C}\right)+\mathbf{K}_{i}(\mathbf{r}\right. \\
& \left.\left.+\mathbf{R}^{C O}(\theta) \mathbf{p}_{i}^{C}-\left(\mathbf{p}_{i}^{O}\right)_{0}\right)\right], \\
\mathbf{J} \boldsymbol{\theta} & =\mathbf{M}_{c}+\sum_{i=1}^{3}\left\{\mathbf{p}_{i}^{C} \times\left[\mathbf{C}_{i}\left(\dot{\mathbf{r}}+\mathbf{R}^{C O}(\theta) \dot{\mathbf{p}}_{i}^{C}\right)\right.\right. \\
& \left.\left.+\mathbf{K}_{i}\left(\mathbf{r}+\mathbf{R}^{C O}(\theta) \mathbf{p}_{i}^{C}-\left(\mathbf{p}_{i}^{O}\right)_{0}\right)\right]\right\},
\end{aligned}
$$

where $\mathbf{R}^{\mathrm{CO}}(\theta)$ is the direction cosine matrix from the coordinate system $\sum C$ to the coordinate system $\sum O, \mathbf{p}_{i}^{\mathrm{O}}$ is the current position, and $\left(\mathbf{p}_{i}^{O}\right)_{0}$ is the initial position of the $i$ th isolator in the global inertial coordinate system.

\section{Design of AHC Controller}

4.1. MIMO to SISO. To solve the problem of multiple DOFs isolation in AVIS, a transformation from MIMO (Multiple-Input Multiple-Output) to SISO (Single-Input SingleOutput) is carried out. The advantage is that the active control parameters of each DOF can be adjusted separately and the optimal control effect of each DOF can be achieved independently. The principle diagram of signal conversion from MIMO to SISO is illustrated in Figure 7.

The signal conversion process is as follows: physical axis coordinate signals (MI) measured by six feedback sensors are converted to six logical axis coordinate signals (SI) by the transform matrix. After meeting with the logic axis coordinate signals (SI) measured by three feedforward sensors, mixed SI signals are delivered to SISO controller. In the SISO controller, the active control of six DOFs is carried out independently. The six logical axis coordinate output signals (SO) from SISO controller are converted to six physical axis coordinate signals (MO) which are used to drive VCMs of different isolators.

4.2. SISO AHC Controller. The overall block diagram of AHC controller is shown in Figure 8. The diagram contains a position loop and a velocity loop. The position loop using PID controller with a low-pass filter is employed for position control, which means the air spring is inflated to the working position and to maintain a stable state. The velocity loop combines a feedback loop with a bandpass filter and a feedforward loop with a bandpass filter also. The absolute velocity sensor is adopted in two loops. Different is the case that the sensor of the feedback loop is responsible for measuring the vibration signal of the payload platform, while the sensor of the feedforward loop is responsible for the base platform.

Because the force and the velocity should be 180 degrees out of phase, the velocity-force feedback should be negative which can make the output play a role in the opposite of the input and reduce the system error between the output and the target. Finally, the control system will tend to be stable in this condition.

4.2.1. Sky-Hook Damping Technology in FBAC. Passive vibration isolation system can reduce high frequency vibration, but for the low frequency resonance peak, the signal is 


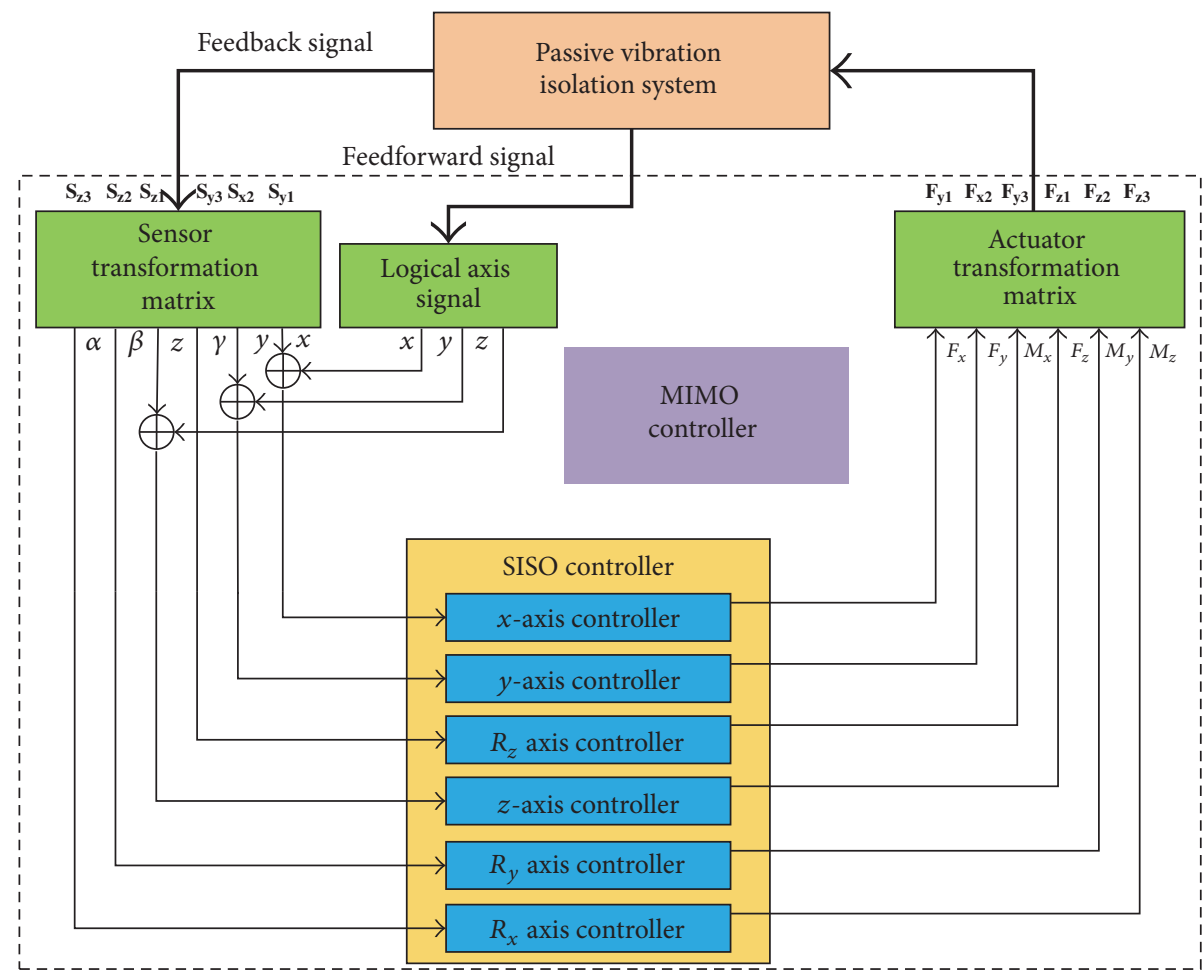

FIGURE 7: The principle diagram of MIMO to SISO.

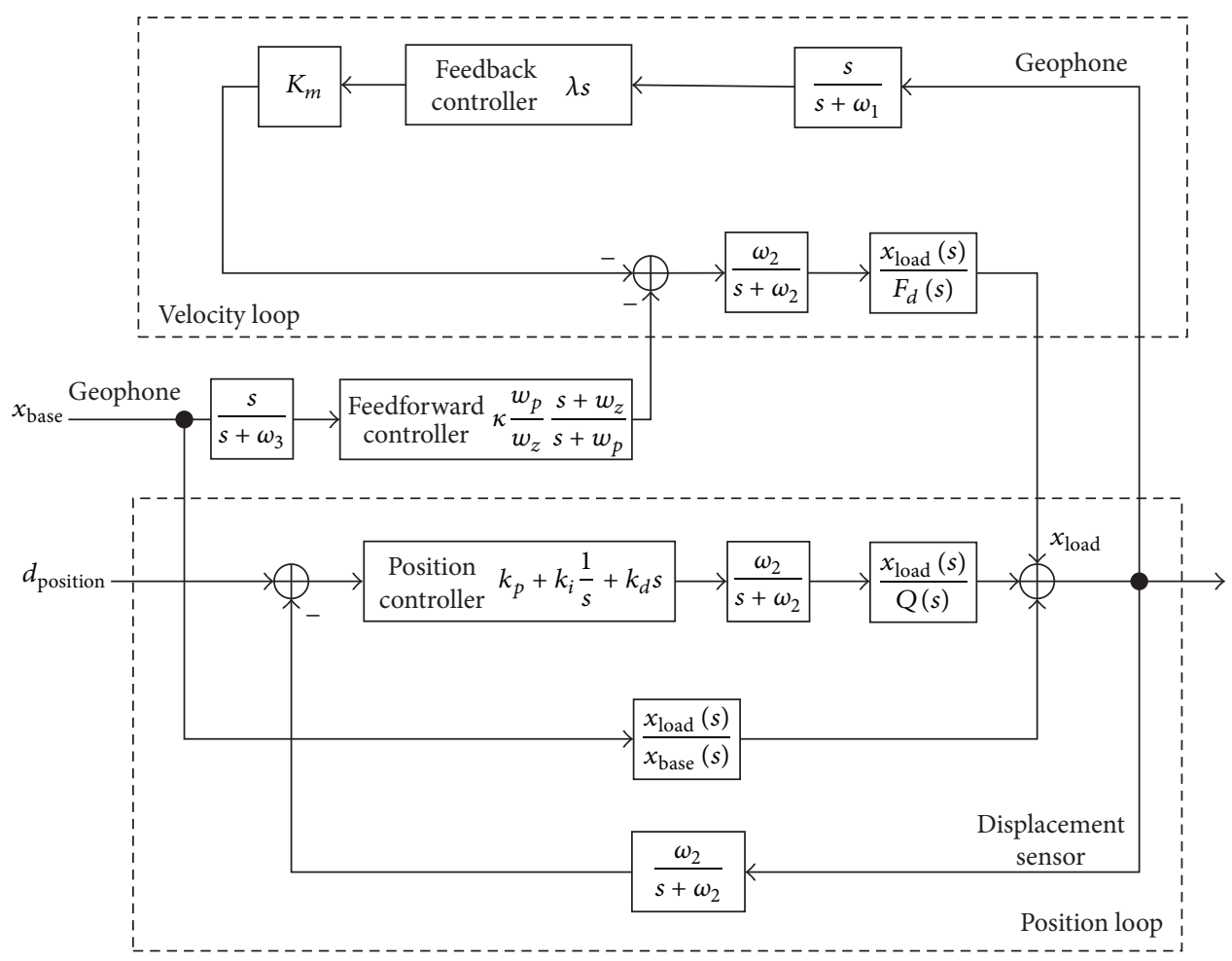

FIGURE 8: The control overall block diagram. 


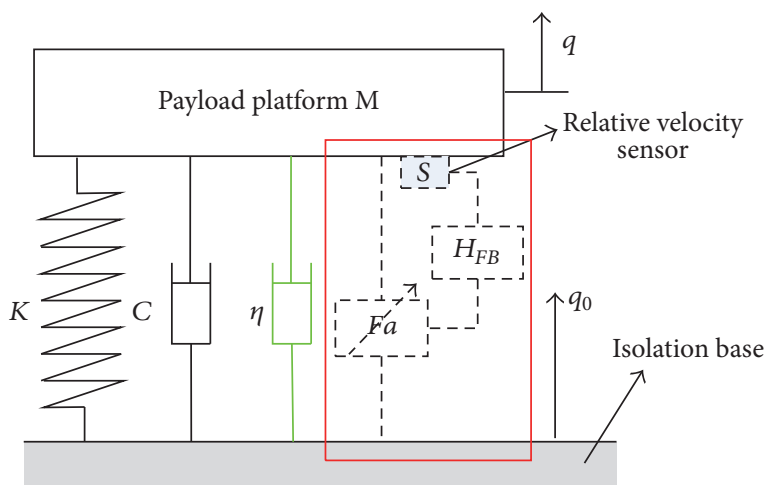

(a)

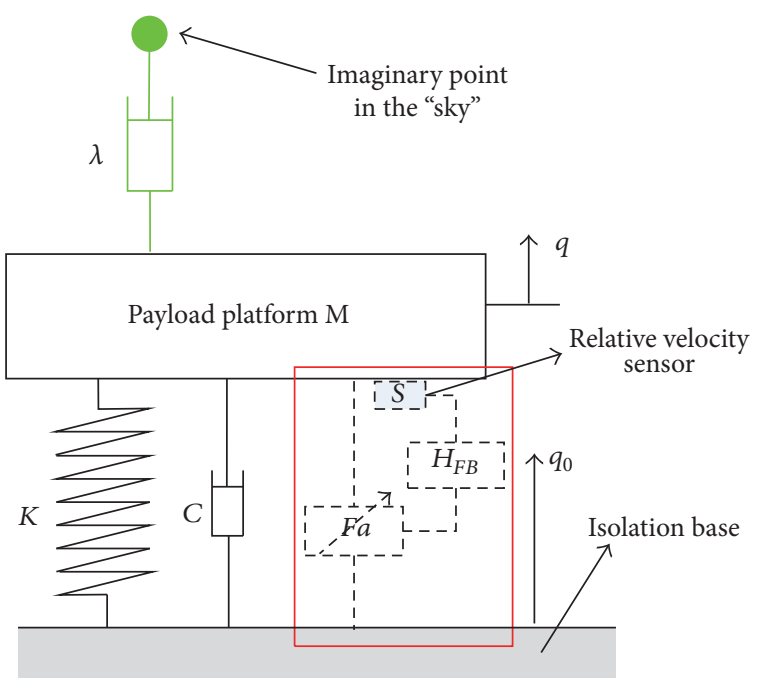

(b)

FIGURE 9: The equivalent schematic diagram of single DOF with velocity-force negative feedback: (a) equivalent relative velocity feedback system and (b) equivalent absolute velocity feedback system.

amplified. Hence, an active feedback control technology can effectively address the problem of amplitude amplification in the resonance peak. Compared with a relative damping feedback control system, sky-hook active control technology which is implemented with an absolute damping feedback control system can effectively overcome the contradiction between low frequency vibration isolation performance and high frequency vibration attenuation. The schematic diagram of a single DOF AVIS with velocity-force negative feedback is approximately equivalent to the situation of Figure 9. Within the red line box in Figure 9 is the complete active control closed loop. The green line means the equivalent damper.

According to Figure 9(a), a damper (green line) placed between isolation base and payload works exactly as a relative velocity-force negative feedback passive control system. The relative velocity sensor, the force generating element (actuator), and the control law (negative proportional velocity-force feedback) are placed in the relative damper. The sensor and the actuator are collocated. An AVIS with relative velocity feedback (in the red line box) does exactly the job of this relative passive damper. It uses a real relative velocity sensor, a real actuator, and a real electrically closed loop with negative proportional velocity-force feedback (with the gain $\eta$ ).

According to Figure 9(b), a damper (green line) placed between a fixed imaginary point in the "sky" and payload works exactly as an absolute velocity-force negative feedback passive control system. The absolute velocity sensor, the force generating element (actuator), and the control law (negative proportional velocity-force feedback) are placed also in the absolute damper. The sensor and the actuator are also collocated. An AVIS with absolute velocity feedback (in the red line box) should do exactly the job of this absolute passive damper. It uses a real absolute velocity sensor, a real actuator, and a real electrically closed loop between with negative proportional velocity-force feedback (with the gain $\lambda)$.
According to Newton-Euler equation, the dynamic equations of the AVIS with relative or absolute velocity negative feedback are written as

$$
\begin{aligned}
& M \ddot{q}+c\left(\dot{q}-\dot{q}_{0}\right)+k\left(q-q_{0}\right)+F_{\mathrm{RV}}=0, \\
& F_{\mathrm{RV}}=\eta\left(\dot{q}-\dot{q}_{0}\right), \\
& M \ddot{q}+c\left(\dot{q}-\dot{q}_{0}\right)+k\left(q-q_{0}\right)+F_{\mathrm{AV}}=0, \quad F_{\mathrm{AV}}=\lambda \dot{q},
\end{aligned}
$$

where $M$ is the mass of payload, $c$ is the equivalent damping, $k$ is the equivalent stiffness, $q$ is the displacement of payload, $q_{0}$ is the displacement of base, $F_{\mathrm{RV}}$ is the force of actuator in relative velocity negative feedback control, $F_{\mathrm{AV}}$ is the force of actuator in absolute velocity negative feedback control, $\eta$ is the gain of relative velocity negative feedback control, and $\lambda$ is the gain of absolute negative feedback control.

The transfer function of a single DOF AVIS with relative or absolute velocity negative feedback control can be expressed as

$$
\begin{aligned}
T_{O}(s) & =\frac{Q(s)}{Q_{0}(s)}=\frac{(c+\eta) s+k}{M s^{2}+(c+\eta) s+k} \\
& \approx \frac{\eta s+k}{M s^{2}+\eta s+k}, \\
T_{C}(s) & =\frac{Q(s)}{Q_{0}(s)}=\frac{c s+k}{M s^{2}+(c+\lambda) s+k} \\
& \approx \frac{k}{M s^{2}+\lambda s+k} .
\end{aligned}
$$

Because the equivalent damping of the structure is far less than the sky-hook damping, the system damping $c$ is neglected.

Depending on (13)-(14), the simulation curves with different control ways are described in Figure 10. It can be seen that 


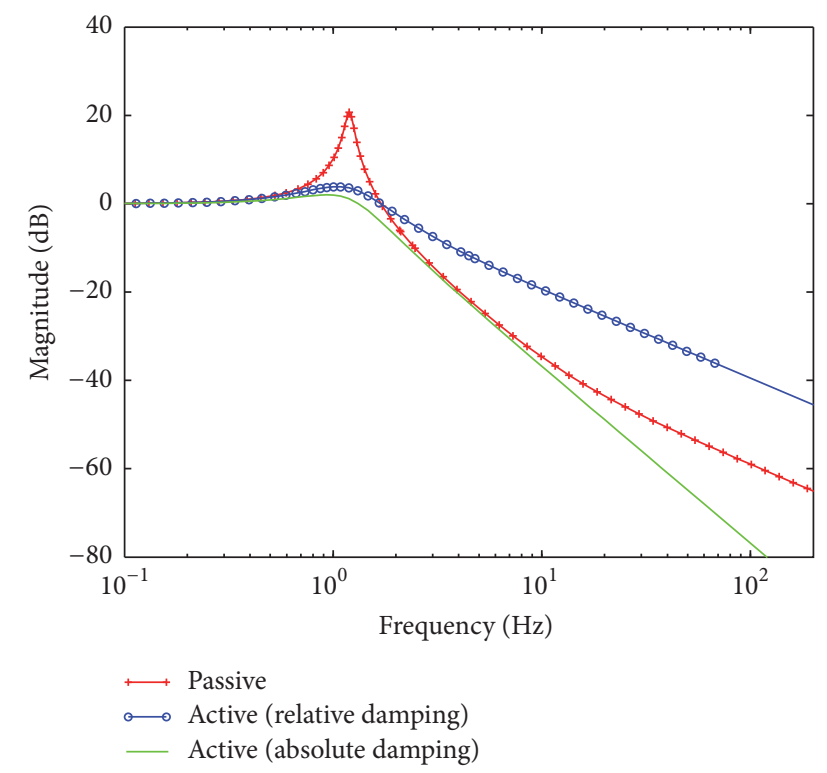

FIGURE 10: The simulation curves of transmissibility with different control ways: (a) the red line for passive with $M=3000 \mathrm{~kg}$ and $k=$ $1.7 e 5 \mathrm{~N} / \mathrm{m}$, (b) the blue line for relative velocity negative feedback with $\eta=20$, and (c) the green line for absolute velocity negative feedback $\lambda=20$.

relative velocity feedback can also reduce the resonance peak amplitude of the vibration system. However, the attenuation rate in high frequency is only $20 \mathrm{~dB} /$ decade, which cannot reach $40 \mathrm{~dB} /$ decade achieved by absolute velocity feedback control system.

4.2.2. LLPC Control Technology in FFAC. The major characteristics of the lead-lag compensator are the constant attenuation of magnitude at high frequencies and the zero phase shift at high frequencies. The large negative phase shift that is seen at intermediate frequencies is undesired but unavoidable. Proper design of the compensator requires placing the compensator pole and zero appropriately so that the benefits of the magnitude attenuation are obtained without the negative phase shift causing problems.

(a) The Structure of Phase Compensator. Based on the traditional phase compensator, the structure of lead-lag phase compensator can be expressed as

$$
G_{11}(s)=\kappa \frac{\tau s+1}{\alpha \tau s+1}=\kappa \frac{w_{p}}{w_{z}} \frac{s+w_{z}}{s+w_{p}},
$$

where $w_{p}$ is the pole frequency and $w_{z}$ is the zero frequency and $\kappa$ is gain. The gain is used to satisfy the steady-state error specification.

Defining the number of open loop poles that are located at $s=0$ to be the system type $N$ and restricting the reference input signal to having Laplace transforms of the equation $R(s)=A / s^{q}$, the gain, steady-state error, and error constant

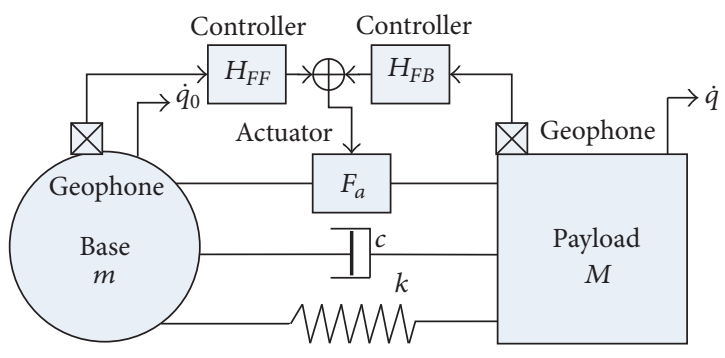

FIGURE 11: Schematic diagram of vibration isolation for absolute velocity feedback control and lead-lag phase compensation feedforward control.

are (assuming that the closed loop system is bounded-input, bounded-output stable)

$$
\begin{aligned}
\kappa & =\frac{e_{\text {ss_plant }}}{e_{\text {ss_specified }}}=\frac{\kappa_{x \_ \text {required }}}{\kappa_{x \_ \text {plant }}}, \\
e_{\text {ss }} & =\lim _{s \rightarrow 0}\left[\frac{A s^{N+1-q}}{s^{N}+\kappa_{x}}\right] \\
\kappa_{x} & =\lim _{s \rightarrow 0}\left[s^{N} G_{11}(s)\right],
\end{aligned}
$$

where $e_{s s}$ is the steady-state error for a particular type of input, such as step or ramp, and $\kappa_{x}$ is the corresponding error constant of the system.

For $N=0$, the steady-state error for a step input $(q=1)$ is $e_{\mathrm{ss}}=A /\left(1+\kappa_{x}\right)$. For $N=0$ and $q>1$, the steady-state error is infinitely large. For $N>0$, the steady-state error is $e_{\mathrm{ss}}=A / \kappa_{x}$ for the input type that has $q=N+1$. If $q<N+1$, the steady-state error is 0 , and if $q>N+1$, the steady-state error is infinite.

(b) The Model of LLPC. The schematic diagram of a single DOF AVIS with absolute velocity feedback control and LLPC feedforward control is shown in Figure 11.

According to Newton-Euler equation, the Laplace transform of dynamic equation can be expressed as

$$
\begin{gathered}
M s^{2} q(s)+c s\left[q(s)-q_{0}(s)\right]+k\left[q(s)-q_{0}(s)\right] \\
+\lambda s q(s)+\kappa \frac{w_{p}}{w_{z}} \frac{s+w_{z}}{s+w_{p}} s q_{0}(s)=0,
\end{gathered}
$$

where $M$ is the mass of payload, $c$ is the equivalent damping, $k$ is the equivalent stiffness, $q$ is the displacement of payload, $q_{0}$ is the displacement of base, $\lambda$ is the gain of feedback control, and $\kappa$ is gain of feedforward control.

The closed loop transfer function of a single DOF AVIS with absolute velocity feedback control and LLPC feedforward control can be organized as 


$$
\begin{aligned}
T(s) & =\frac{Q(s)}{Q_{0}(s)}=\frac{\left(c-\kappa\left(w_{p} / w_{z}\right)\left(\left(s+w_{z}\right) /\left(s+w_{p}\right)\right)\right) s+k}{M s^{2}+(c+\lambda) s+k} \\
& =\frac{\left(c w_{z}-\kappa w_{p}\right) s^{2}+w_{z}\left(k+w_{p}-\kappa w_{p}\right) s+k w_{z} w_{p}}{M w_{z} s^{3}+w_{z}\left(M w_{p}+c+\lambda\right) s^{2}+w_{z}\left[w_{p}(c+\lambda)+k\right] s+k w_{z} w_{p}} .
\end{aligned}
$$

(c) The Stability Margin of AVIS. The purpose of phase compensator design in the frequency domain generally is to satisfy specifications on steady-state accuracy and phase margin. There may also be a specification on the gain crossover frequency or closed loop bandwidth. A phase margin specification can represent a requirement on relative stability due to pure time delay in the system, or it can represent desired transient response characteristics that have been translated from the time domain into the frequency domain $[7,10,19]$.

The phase margin and gain margin can be expressed, respectively, as

$$
\begin{aligned}
& \varphi=180^{\circ}+\angle G_{\mathrm{pa}}(j \omega) H_{\mathrm{co}}(j \omega), \quad \varphi(\mathrm{deg})>0, \\
& h=\frac{1}{\left|G_{\mathrm{pa}}(j \omega) H_{\mathrm{co}}(j \omega)\right|}, \quad h(\mathrm{~dB})>0,
\end{aligned}
$$

where $G_{\mathrm{pa}}(\mathrm{s})$ is the transfer function of passive vibration isolation system and $H_{\mathrm{co}}(s)$ is the transfer function of AHC controller.

According to (15) and (20)-(21), the bode diagram of phase compensator is illustrated in Figure 12. When discrete parameters are taken, the phase margin and gain margin of bode diagram produce changes accordingly. From Figure 12, it can be seen that the newly introduced value where $h(\mathrm{~dB})$ and $\varphi(\mathrm{deg})$ are positive will lead the system to a more stable trend. On the contrary, the trend is opposite.

(d) The Simulation of Controller. According to (14) and (20), simulation curves with different control ways are described in Figure 13. It can be observed that sky-hook damping active control can reduce the resonance peak amplitude and guarantee the high attenuation rate $(40 \mathrm{~dB} /$ decade) in high frequency simultaneously. Based on sky-hook damping, LLPC can further reduce the amplitude of transmissibility. At the same time, active effective bandwidth is extended from near the natural frequency to the midfrequency region. The phase margin of the system is positive, and gain margin is also further enhanced.

The response curves under different excitation conditions are illustrated in Figure 14. In Figure 14(a), it can be seen that the vibration isolation system is restored to the steadystate after 10s in the condition of the passive state. However, when the active control (AHC) is turned on, the stable-time of the AVIS system is reduced to about $1 \mathrm{~s}$. Figure 14(b) shows that the vibration amplitude is decreased obviously when the active control (AHC) works.

\section{Experimental Results and Discussions}

5.1. Set-Up of Experiments. According to the layout of AVIS, an experimental system of Figure 15 which includes a realtime active control system and a spectrum testing and analysis system is set up in the super-clean lab. In the active control system of Figure 15(b), a control cubicle which consists of the data acquisition board and the driver board is responsible for the input and output of signals, respectively, where the used signals are analog signals. The MCU module is replaced by an NI PXI bus embedded controller (model: PXIE-8135 which consists of an Analog Signal Input Card, PXI-6123, and an Analog Signal Output Card, PXI-6733) which is operated in the form of digital signals.

A vibration generator (model: MB MODAL110) is adopted to simulate the simulated environmental conditions signal that is generated by a signal simulation generator (model: LMS SCADASIII SCM05). At the same time, two servo velocity meters (Model: VSE-15D6) are installed on the base platform and the payload platform, respectively, to collect the vibration signals of the base platform and the residual vibration signal of the payload platform after active control. Finally, the collected data is imported into the vibration acquisition and analysis instruments (model: LMS SCADASIII SCM05) for signal analysis and processing.

5.2. Experimental Results. Figure 16 which illustrates the contrast about the velocity admittance curves of logical axes is mainly adopted for verifying the decoupling and the accuracy of the dynamic model. It can be seen that the decoupling of various DOF is basically achieved. The results make it possible for multi-DOFs active control. In addition to the main resonance peak, there is a coupling peak in the direction of $X$ translation. It may be caused by inadequate decoupling which is generated from the centroid offset without timely compensation and residual effect from $R x$ direction.

In the following experiments, to simulate the vibration criterion of VC-A (the velocity spectrum in $1 / 3$ octave is $50 \mu \mathrm{m} / \mathrm{s}$ ), the excitation signal is set as a random signal that the amplitude voltage is $0.1 \mathrm{~V}$ and the frequency band is $200 \mathrm{~Hz}$ to the power amplifier for the control of vibration generator. In LMS software, the bandwidth of acquisition signal is $200 \mathrm{~Hz}$, spectral lines is 2048 . A linear average is taken for the 20 sets of data. At the same time, the signals are inserted in a force window to facilitate the processing analysis.

On the signal processing technique, the amplitude ratio of vibration response (sensor signals measured on the payload 
TABLE 2: The results of transmissibility in $X / Y / Z$.

\begin{tabular}{|c|c|c|c|c|c|c|}
\hline \multirow{2}{*}{ Direction } & \multicolumn{2}{|c|}{$X$} & \multicolumn{2}{|c|}{ Y } & \multicolumn{2}{|c|}{$Z$} \\
\hline & $1.20 \mathrm{~Hz}$ & $10 \mathrm{~Hz}$ & $1.19 \mathrm{~Hz}$ & $10 \mathrm{~Hz}$ & $1.17 \mathrm{~Hz}$ & $10 \mathrm{~Hz}$ \\
\hline Passive (dB) & 15.67 & -31.90 & 20.79 & -31.44 & 19.98 & -34.78 \\
\hline Active (feedback only) (dB) & -5.70 & -34.14 & -2.27 & -37.74 & -0.85 & -36.38 \\
\hline Active (AHC) (dB) & -8.95 & -47.58 & -8.38 & -44.64 & -3.60 & -41.14 \\
\hline Decrease $(\mathrm{dB})$ & 24.62 & 15.68 & 29.17 & 13.20 & 23.58 & 6.36 \\
\hline Attenuation rate (\%) & 64.31 & 99.58 & 61.89 & 99.41 & 33.93 & 99.12 \\
\hline Active effective bandwidth $(\mathrm{Hz})$ & \multicolumn{2}{|c|}{$0.25 \sim 32.82$} & \multicolumn{2}{|c|}{$0.41 \sim 45.74$} & \multicolumn{2}{|c|}{$0.23 \sim 14.38$} \\
\hline
\end{tabular}
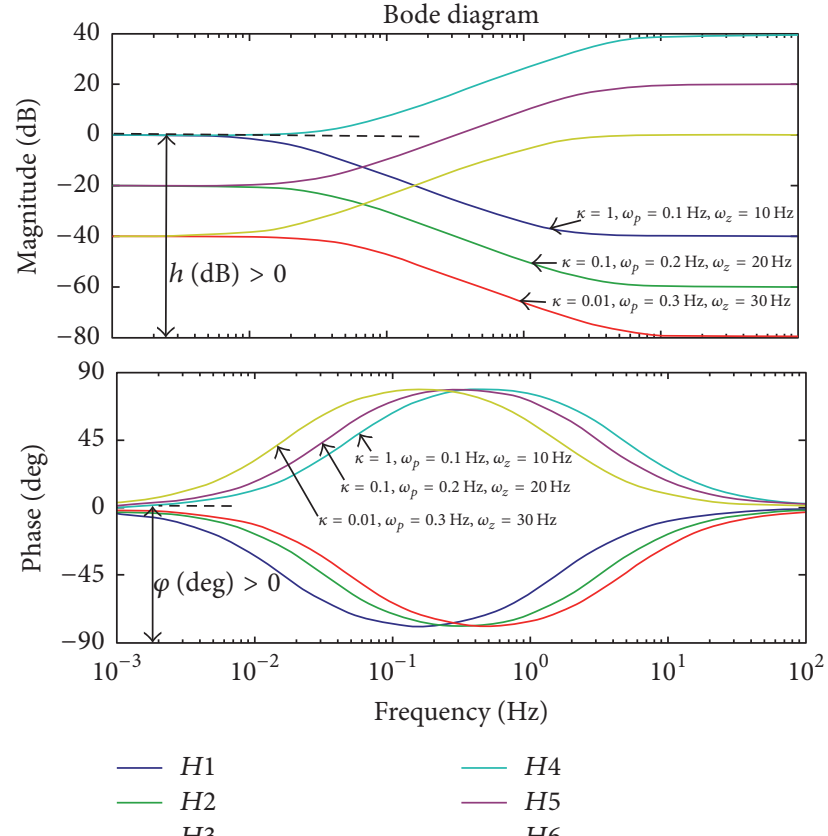

FIGURE 12: The bode diagram of phase compensator.

platform) to excitation input (sensor signals measured on the base platform) is subjected to Fourier transform for the formation of transmissibility curves. Under the different conditions of passive and active, the vibration transmissibility curves are shown in Figure 17, respectively. Detailed curve data of different directions are listed in Table 2 correspondingly.

The auto-power spectrum curves and the cross-power spectrum curves of the response signal and excitation signal are shown in Figures 18 and 19 independently. These power spectrum curves are estimated by means of the average smooth periodogram method. The experimental results in the time domain are presented in Figure 20 and Table 3 below for the comparison of different conditions.

When in the open loop (passive) state, in $X / Y / Z$ directions, the red solid line of Figure 17 shows that the resonance peak frequency of the vibration isolation system is $1.20 \mathrm{~Hz} / 1.19 \mathrm{~Hz} / 1.17 \mathrm{~Hz}$, respectively, listed in Table 2, and the resonance peak amplitude is $15.67 \mathrm{~dB} / 20.79 \mathrm{~dB} / 19.98 \mathrm{~dB}$. From Table 2, it can be seen that the amplitude in $10 \mathrm{~Hz}$ is $-31.90 \mathrm{~dB} /-31.44 \mathrm{~dB} /-34.78 \mathrm{~dB}$, respectively. Ultra-low

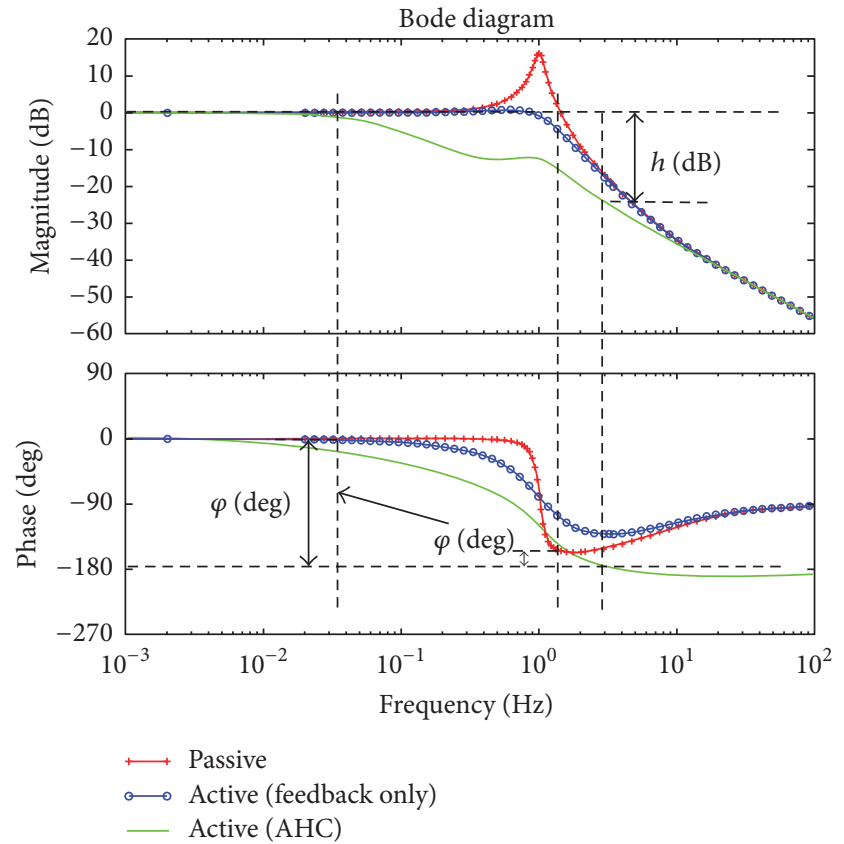

FIGURE 13: The simulation curves of transmissibility with different control ways: (a) the red line for passive with $M=3000 \mathrm{~kg}$ and $k=1.7 e 5 \mathrm{~N} / \mathrm{m}$, (b) the blue line for absolute velocity feedback (skyhook damping) with $\lambda=20$, and (c) the green line for active (AHC: absolute velocity feedback control with $\lambda=20$ and lead-lag phase compensation feedforward control with $\kappa=0.03, w_{p}=0.4 \mathrm{~Hz}$, and $\left.w_{z}=38.5 \mathrm{~Hz}\right)$.

TABLE 3: The time domain signal under different conditions.

\begin{tabular}{|c|c|c|c|}
\hline & Passive & $\begin{array}{c}\text { Active (feedback } \\
\text { only) }\end{array}$ & $\begin{array}{l}\text { Active } \\
\text { (AHC) }\end{array}$ \\
\hline Base $(\mu \mathrm{m} / \mathrm{s})$ & 155 & 155 & 155 \\
\hline Payload $(\mu \mathrm{m} / \mathrm{s})$ & 49 & 12 & 8 \\
\hline $\begin{array}{l}\text { Average vibration } \\
\text { attenuation rate }(\%)\end{array}$ & 68.4 & 92.3 & 94.8 \\
\hline $\begin{array}{l}\text { Payload platform vibration } \\
\text { residual rate }(\%)\end{array}$ & 100 & 24.5 & 16.3 \\
\hline
\end{tabular}

natural frequency of the AVIS mainly owes to the isolators with the structure of positive and negative stiffness in parallel, but the amplitude of the peak is very high in the open loop state. 


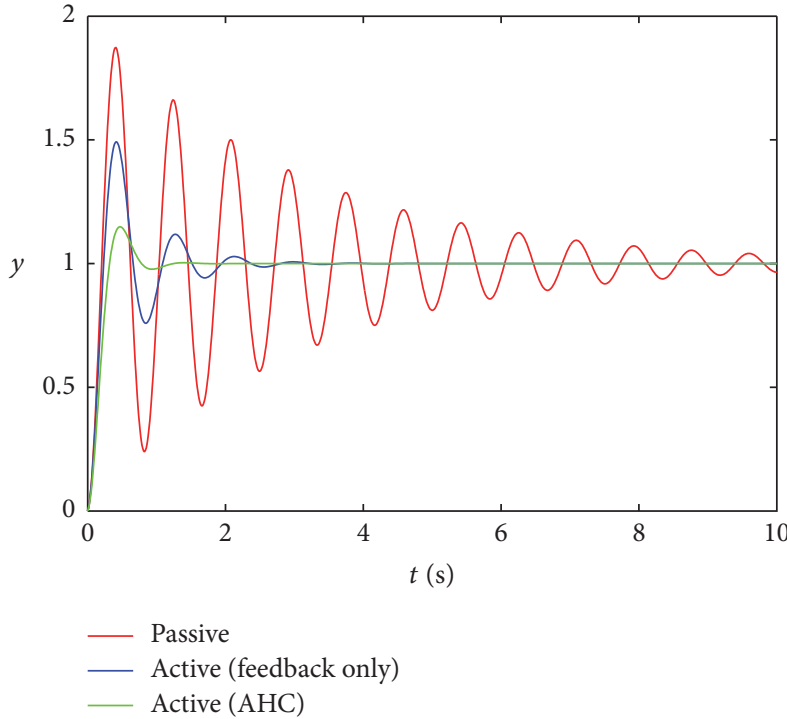

(a)

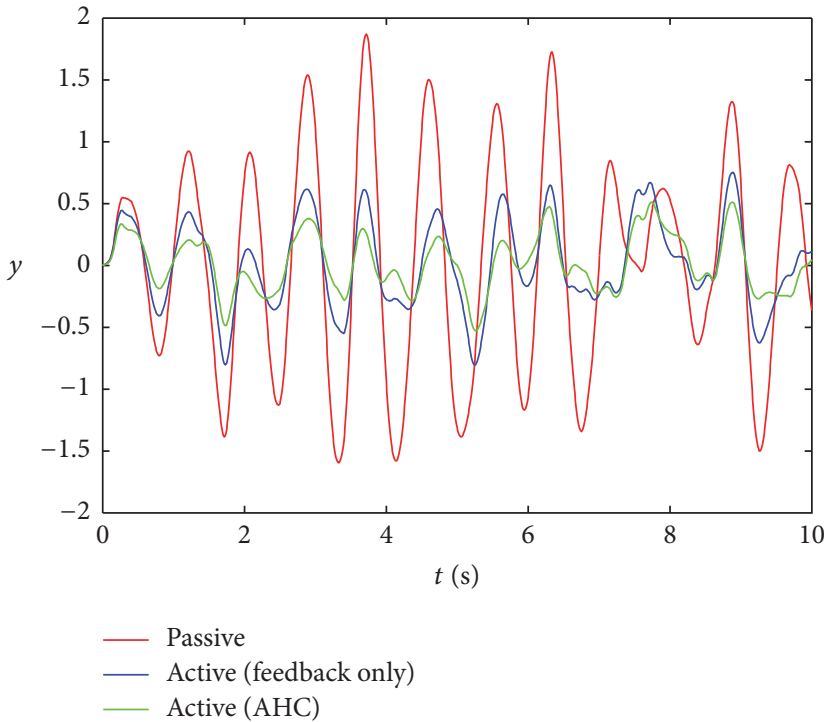

(b)

Figure 14: The response curves under different excitation conditions, the red line for passive with $M=3000 \mathrm{~kg}$ and $k=1.7 e 5 \mathrm{~N} / \mathrm{m}$, the blue line for absolute velocity feedback (sky-hook damping) with $\lambda=20$, and the green line for active (AHC: absolute velocity feedback control with $\lambda=20$ and lead-lag phase compensation feedforward control with $\kappa=0.03, w_{p}=0.4 \mathrm{~Hz}$, and $w_{z}=38.5 \mathrm{~Hz}$ ): (a) impulse response and (b) random response.

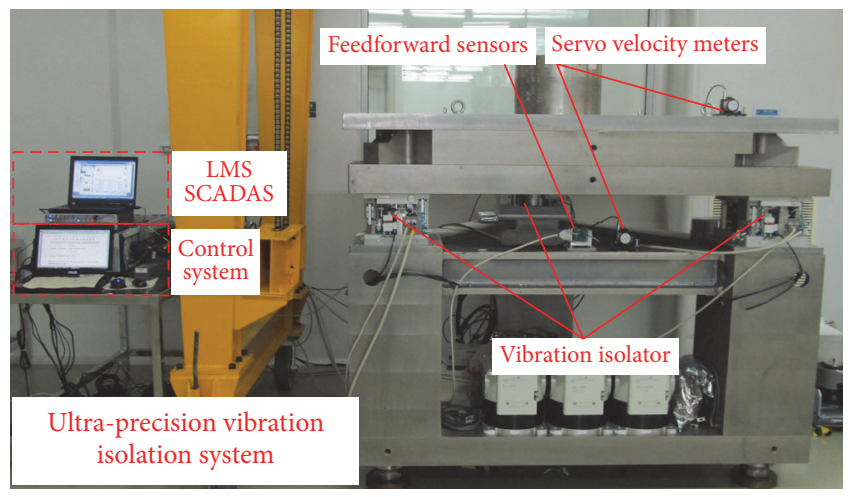

(a)

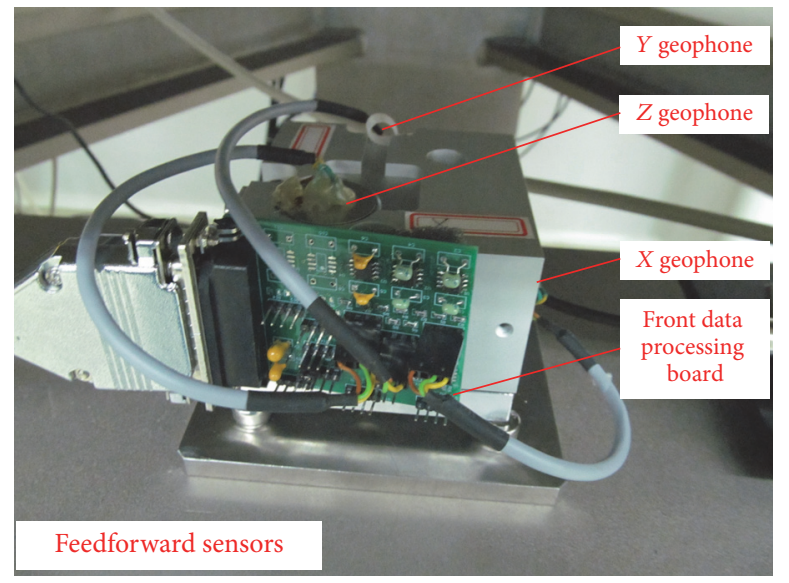

(c)

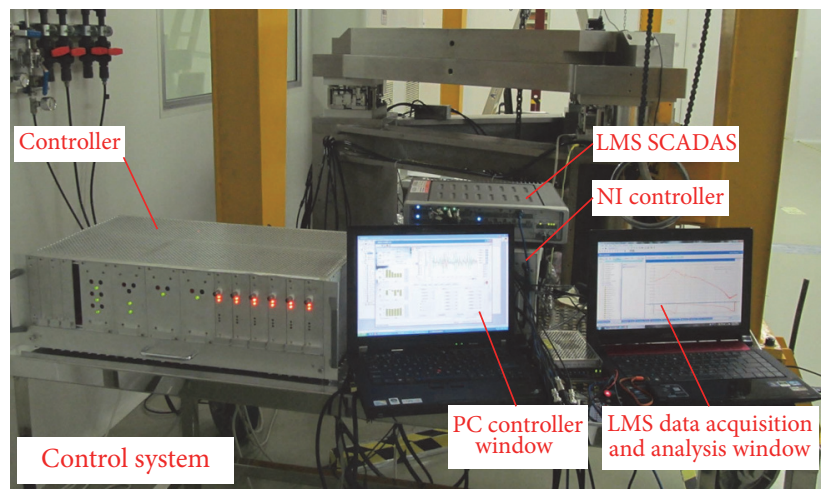

(b)

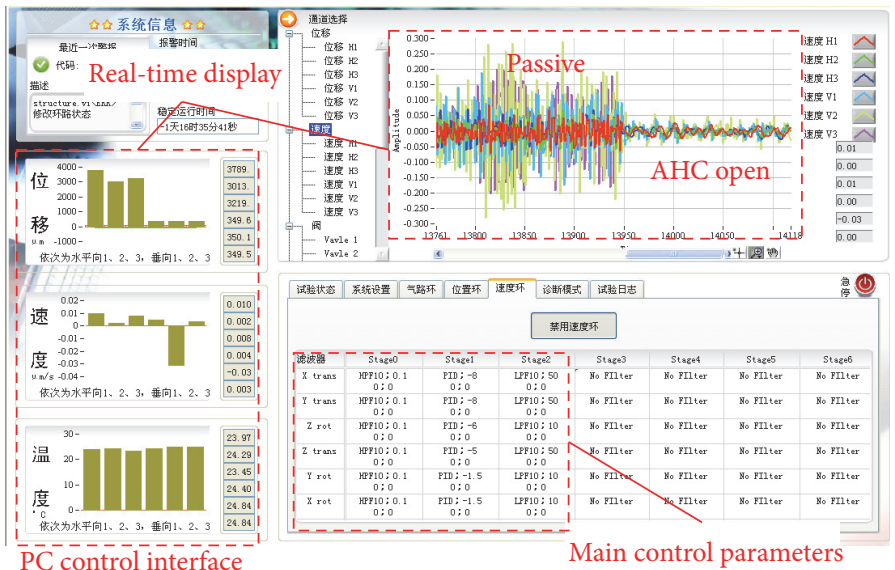

(d)

FIGURE 15: The photo of the ultra-precision vibration isolation experimental system: (a) the layout and installation of the system, (b) the control segment of the system, (c) the feedforward sensor, and (d) the PC control interface. 


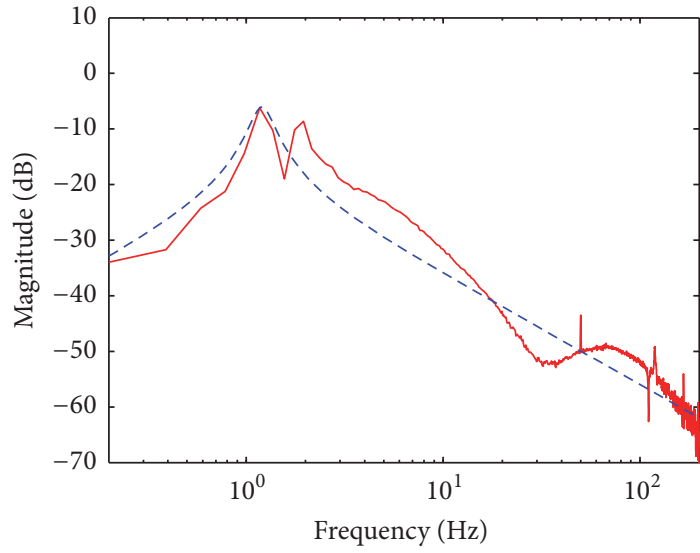

- X-experiment

-.- X-calculation

(a)

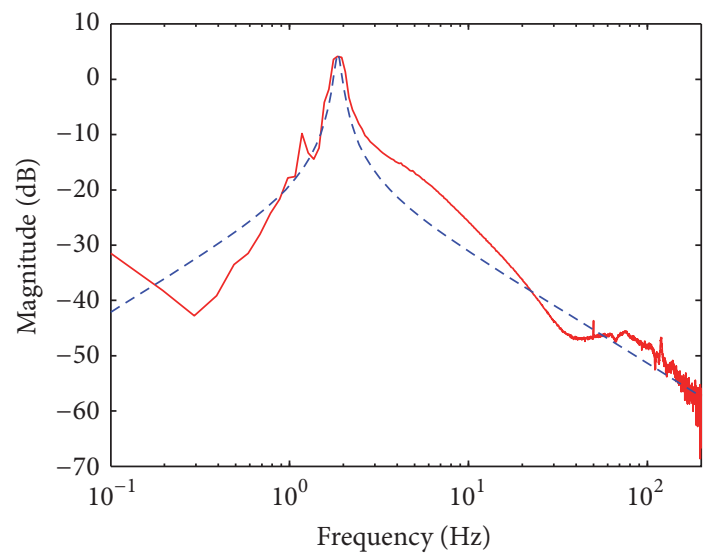

$-R_{z}$-experiment

--- $R_{z}$-calculation

(c)

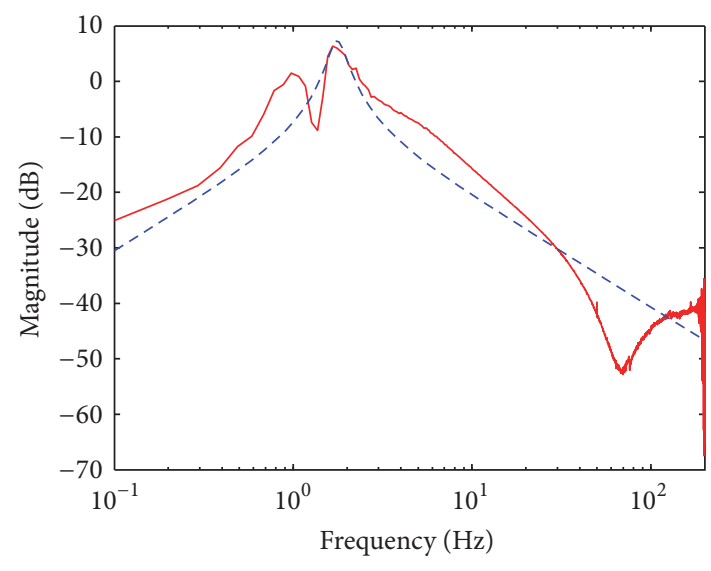

$-R_{y}$-experiment
$---R_{y}$-calculation

(e)

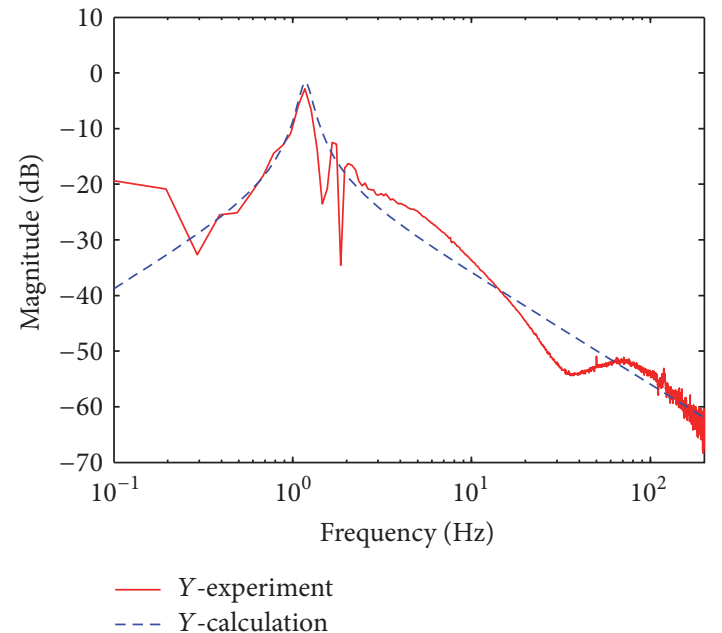

(b)

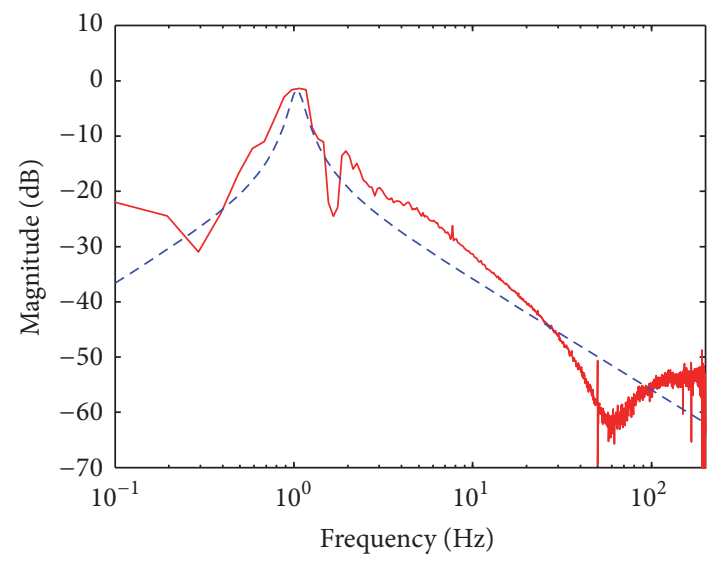

- Z-experiment

-.- Z-calculation

(d)

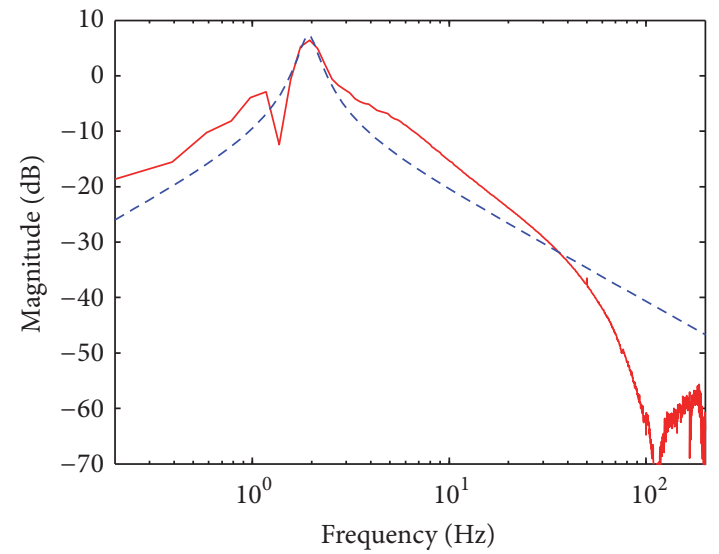

_- $R_{x}$-experiment

(f)

FIGURE 16: The velocity admittance curves of logical axes: (a) $X$ translation, (b) $Y$ translation, (c) $Z$ rotation, (d) $Z$ translation, (e) $Y$ rotation, and (f) $X$ rotation. 


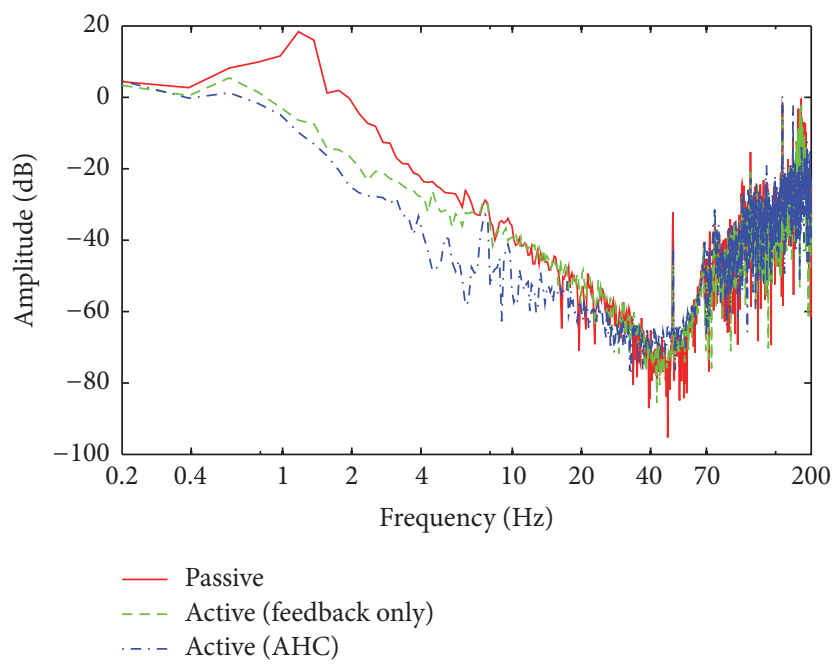

(a)

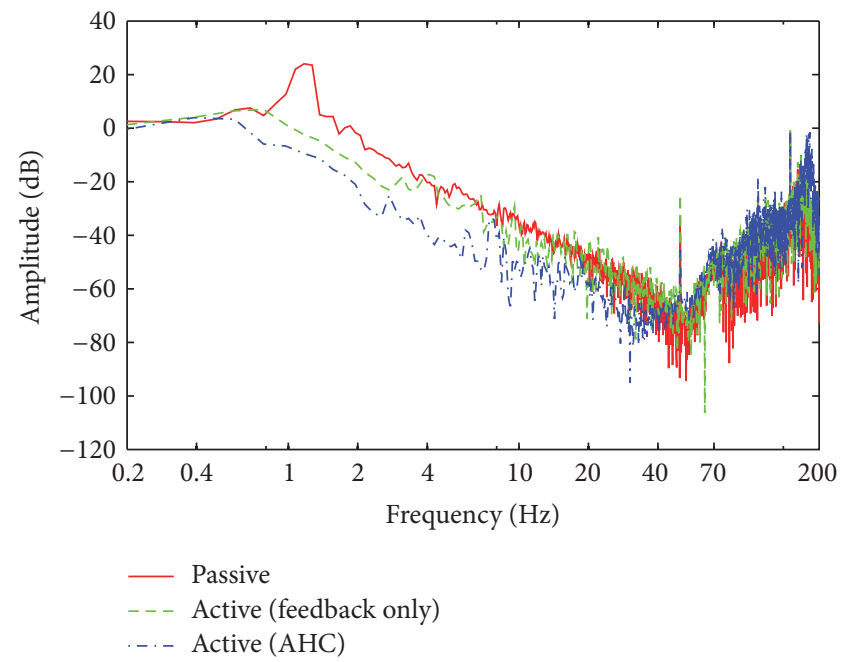

(b)

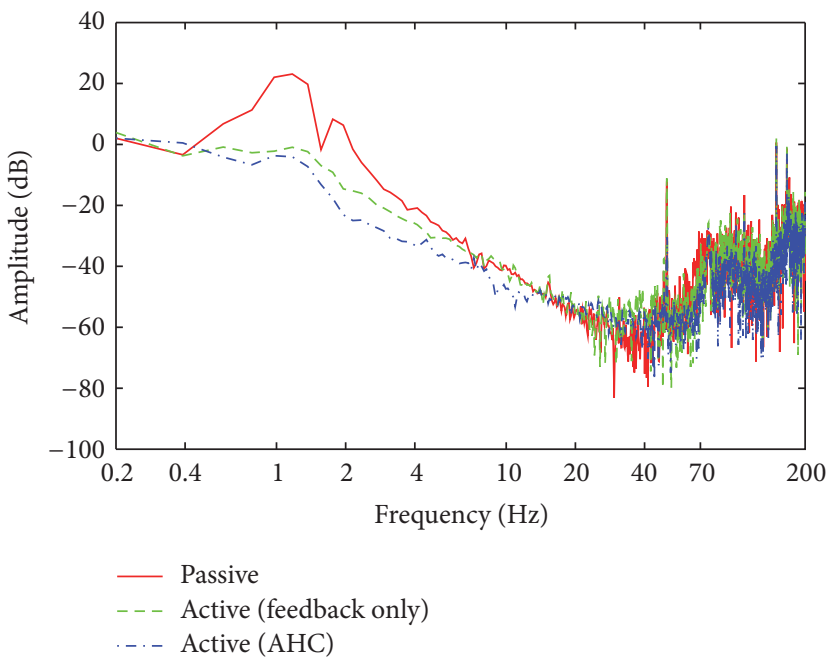

(c)

Figure 17: The vibration transmissibility curves, the red solid line for passive (passive), the blue dashed line for active (absolute velocity feedback control alone, with $\lambda=8$ ), and the green dash and dot line for active (absolute velocity feedback control with $\lambda=8$ and LLPC feedforward control with $\kappa=0.011, w_{z}=0.125 \mathrm{~Hz}$, and $w_{p}=11 \mathrm{~Hz}$ ): (a) $X$, (b) $Y$, and (c) $Z$.

When in the closed loop (sky-hook damping feedback only) state, transmissibility curves of the system are shown as the green dotted line of Figure 17 and in Table 2. From the result, it can be seen that the amplitude of the resonance peak is greatly attenuated, and it is $-5.7 \mathrm{~dB} /-2.27 \mathrm{~dB} /-0.93 \mathrm{~dB}$ in $X / Y / Z$ directions; the amplitude in $10 \mathrm{~Hz}$ is $-34.14 \mathrm{~dB} /-37.74 \mathrm{~dB} /-36.38 \mathrm{~dB}$ respectively. Compared with the open loop, the amplitude of the resonance peak is decreased by $21.37 \mathrm{~dB} / 23.06 \mathrm{~dB} / 20.83 \mathrm{~dB}$; the amplitude in $10 \mathrm{~Hz}$ is reduced by $2.24 \mathrm{~dB} / 6.3 \mathrm{~dB} / 1.6 \mathrm{~dB}$, respectively.

The blue dot line shows the situation in the condition of closed loop (AHC: sky-hook damping feedback and LLPC feedforward) state. Detailed values in Figure 17 are listed in Table 2. In $X / Y / Z$ directions, it can be seen that the amplitude of the resonance peak is further attenuated, and it is only $-8.95 \mathrm{~dB} /-8.38 \mathrm{~dB} /-3.60 \mathrm{~dB}$, respectively; the amplitude in $10 \mathrm{~Hz}$ is $-47.58 \mathrm{~dB} /-44.64 \mathrm{~dB} /-41.14 \mathrm{~dB}$, respectively.
Compared with the open loop, the amplitude of the resonance peak is decreased by $24.62 \mathrm{~dB} / 29.17 \mathrm{~dB} / 23.58 \mathrm{~dB}$; the amplitude in $10 \mathrm{~Hz}$ is reduced by $15.68 \mathrm{~dB} / 13.2 \mathrm{~dB} / 6.36 \mathrm{~dB}$, respectively. The vibration attenuation rate at the resonance peak is $64.31 \% / 61.89 \% / 33.93 \%$ and is $99.58 \% / 99.41 \% / 99.12 \%$ in $10 \mathrm{~Hz}$.

After the AHC, the active effective bandwidth is not only limited to the nearby resonance frequency. And the active effective bandwidth (the value of the last line of Table 2) is extended to the midfrequency region of $0.25 \mathrm{~Hz} \sim$ $32.82 \mathrm{~Hz} / 0.41 \mathrm{~Hz} \sim 45.74 \mathrm{~Hz} / 0.23 \mathrm{~Hz} 14.38 \mathrm{~Hz}$. Simultaneously, AHC algorithm can ensure the high performance of additional frequency range.

From Figure 18, the following results can be drawn. In the passive state, the energy distribution of the payload platform is larger than that of the base platform in the vicinity of the natural frequency, which means that the vibration signal near 


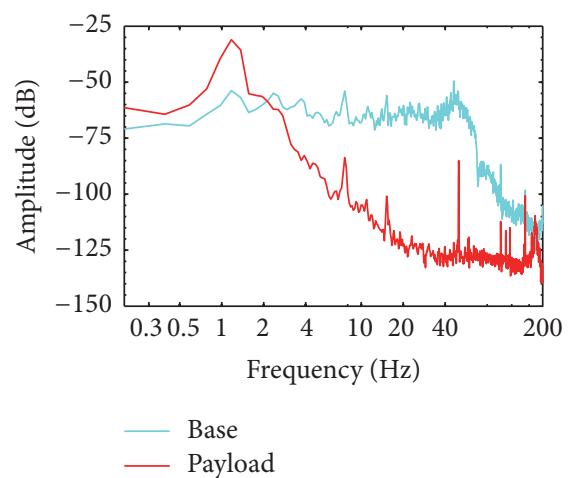

(a)

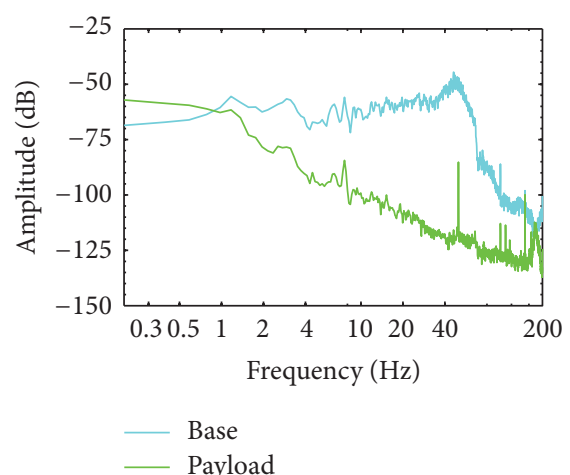

(d)

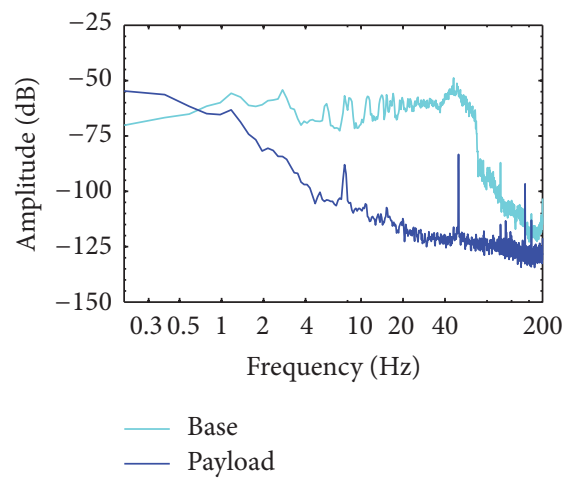

(g)

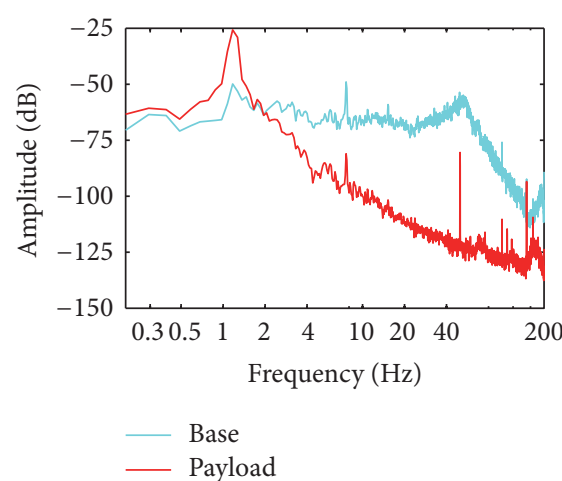

(b)

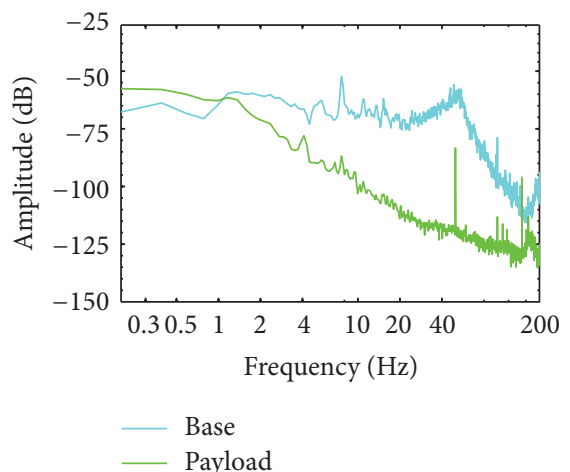

(e)

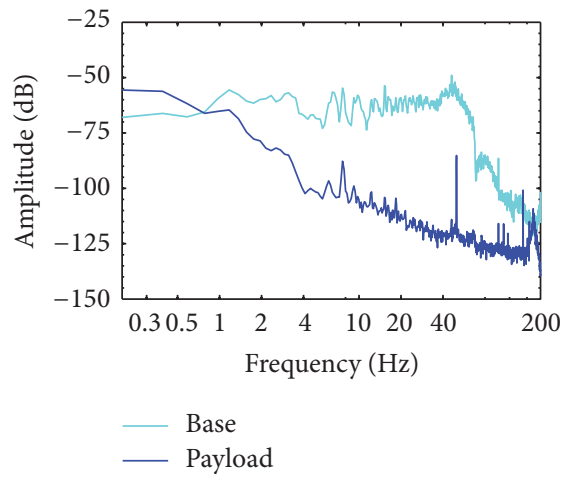

(h)

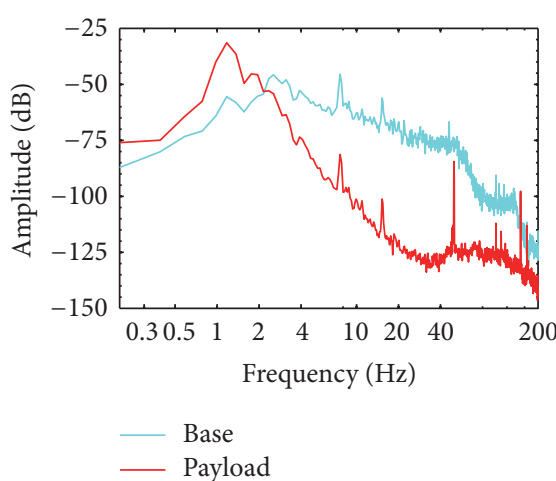

(c)

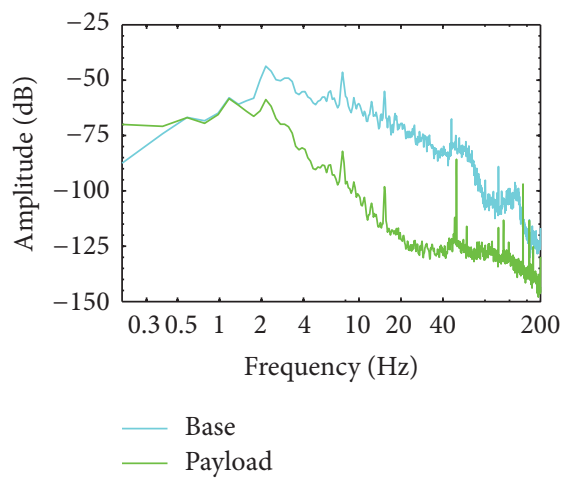

(f)

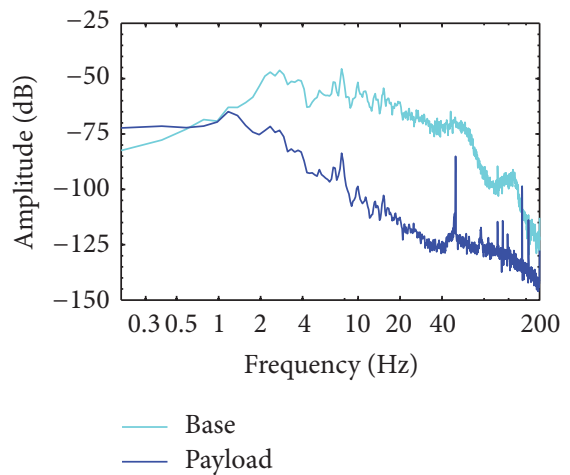

(i)

FIGURE 18: The auto-power spectrum curves: the cyan line for base platform, the red line for payload platform under the passive state, the green line for payload platform under the active (feedback only) state, and the blue line for payload platform under the active (AHC) state: (a) $X$ under passive state, (b) $Y$ under passive state, (c) $Z$ under passive state, (d) $X$ under active (feedback only) state, (e) $Y$ under active (feedback only) state, (f) $Z$ under active (feedback only) state, (g) $X$ under active (AHC) state, (h) $Y$ under active (AHC) state, and (i) $Z$ under active (AHC) state.

the natural frequency is amplified. The energy distribution of the payload platform is significantly smaller than the energy of the base platform in the high frequency band. The passive components play a major role in the high frequency band. In the active closed loop state, this situation has been improved. The energy distribution of the payload platform is close to and gradually less than that of the base platform in the vicinity of the natural frequency, which means that active control begins to work. Compared with the green line (Feedback only), the blue line (AHC) shows the further attenuation of energy distribution.
In Figure 19, the situation is similar to the expression of Figure 18. Compared with the passive state (red line), the energy of the active state (feedback only, green line) is significantly decreased on the resonance frequency point, the energy of the active state (AHC, blue line) is further decreased, and the three directions show a similar situation.

In time domain, the excitation signal of the base platform is about $155 \mu \mathrm{m} / \mathrm{s}$. In the open loop (passive) state, the time domain signal of the payload platform acquired by the geophone is approximate to $\pm 49 \mu \mathrm{m} / \mathrm{s}$ in Figure 20 and Table 3. Compared with the base platform, the average 


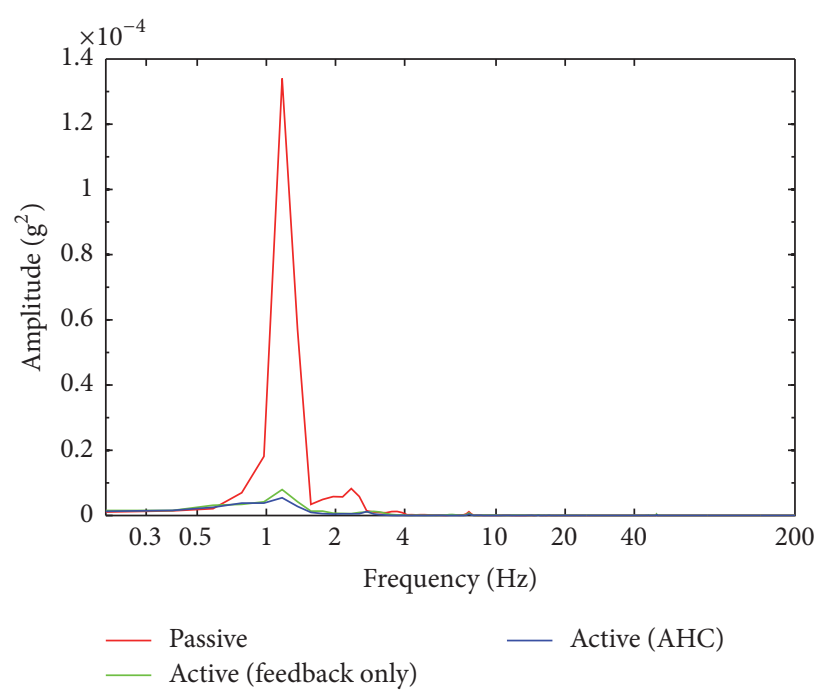

(a)

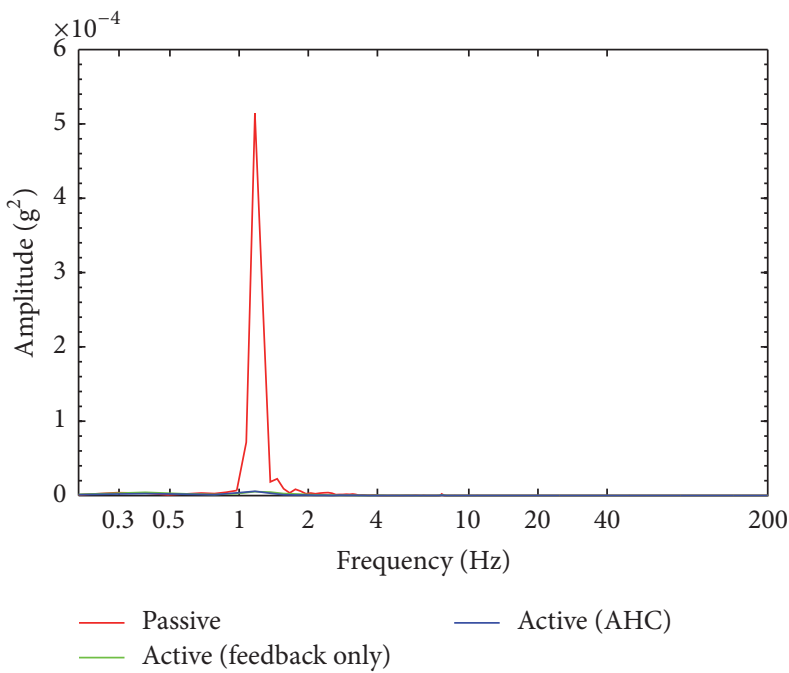

(c)

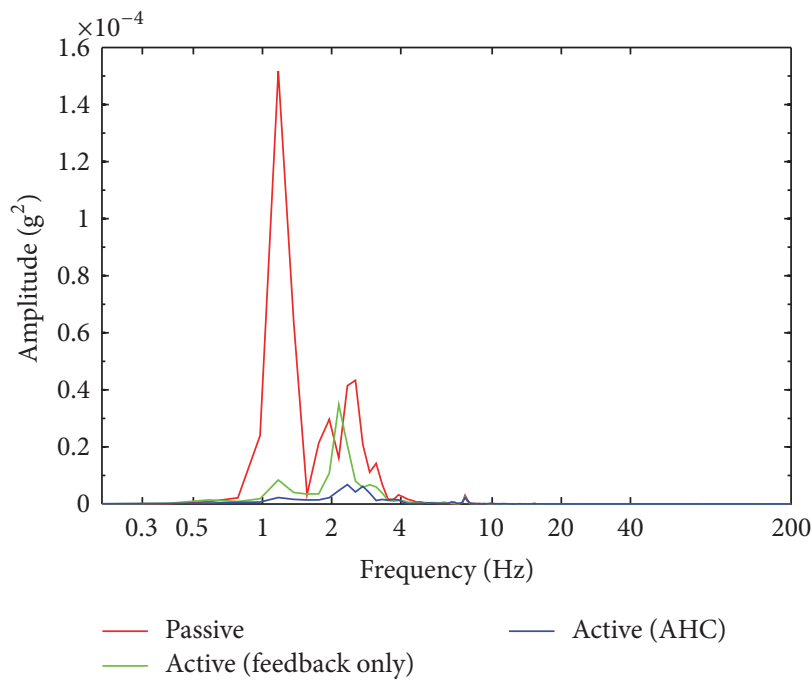

(e)

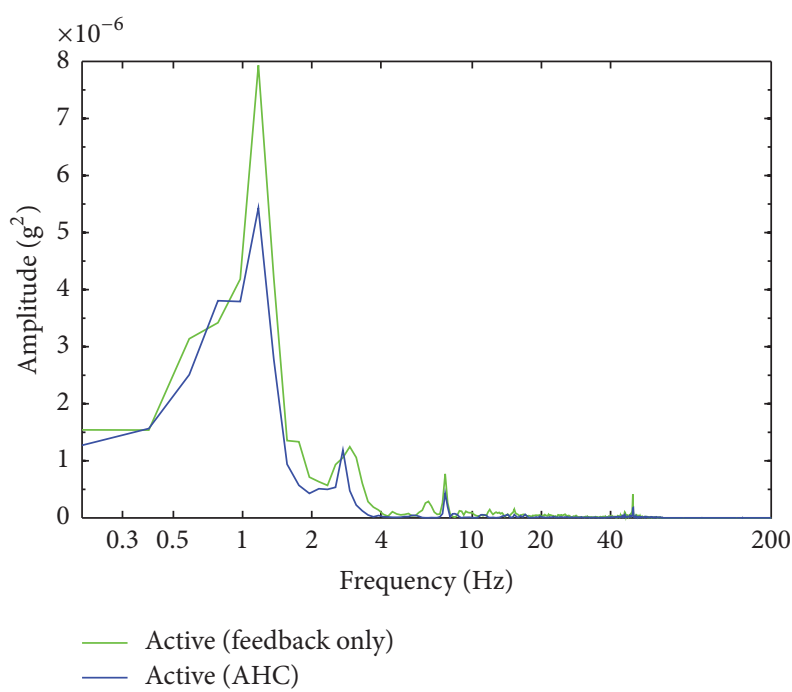

(b)

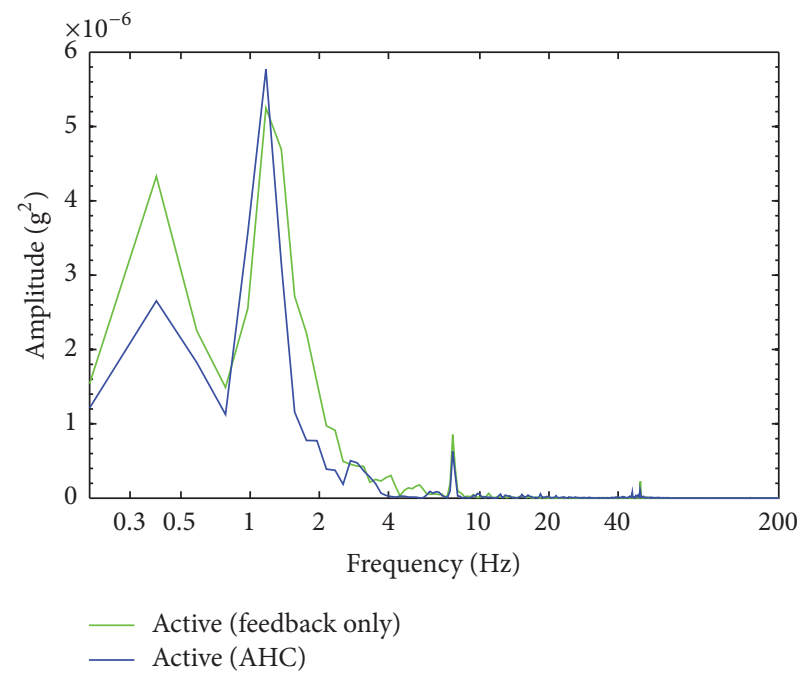

(d)

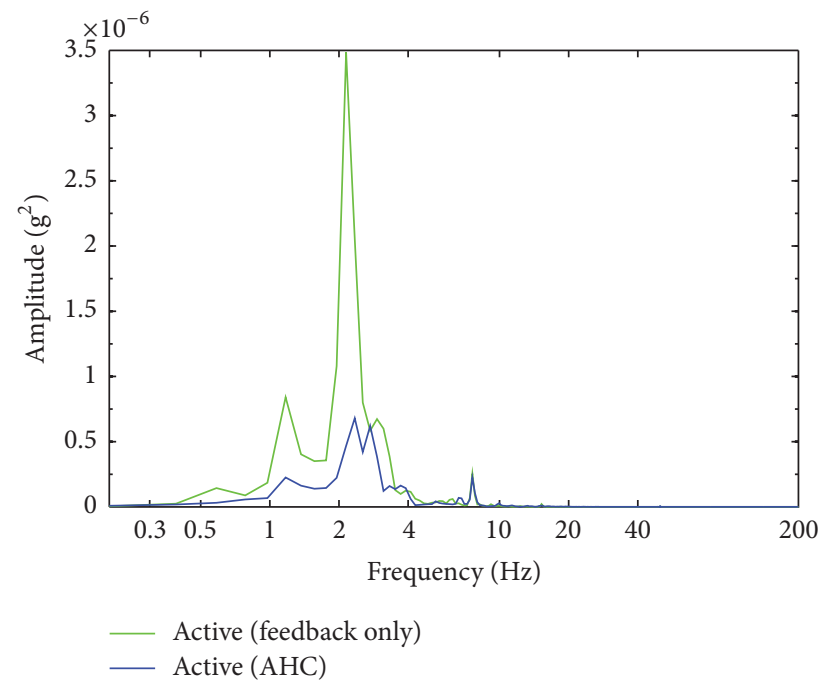

(f)

FIGURE 19: The cross-power spectrum curves: the red line for passive, the green line for active (feedback only), and the blue line for active (AHC). (a) $X$ direction under different conditions, (b) the enlarged view of some curves in (a), (c) $Y$ direction under different conditions, (d) the enlarged view of some curves in (c), (e) $Z$ direction under different conditions, and (f) the enlarged view of some curves in (e). 


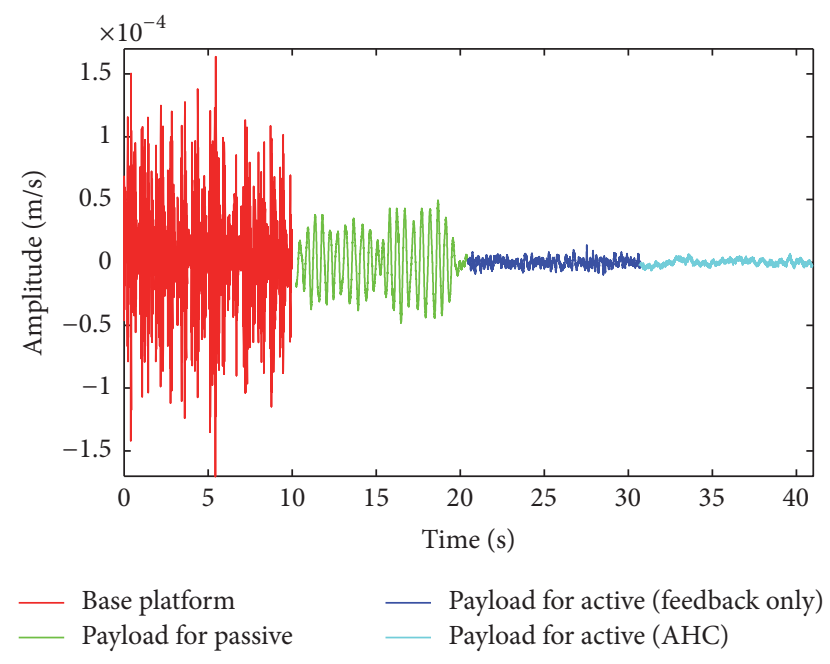

FIGURE 20: The time-domain signal of payload, the red line for base platform, the green line for payload platform in passive state, the blue line for payload platform in active (feedback only) state, and the cyan line for payload platform in active (AHC) state.

vibration attenuation rate is $68.4 \%$. Still from Figure 20 and Table 3, it also can be seen that the time domain signal of the payload platform is only approximate for $\pm 12 \mu \mathrm{m} / \mathrm{s}$ in the closed loop (feedback only) state. The vibration amplitude in the time domain is greatly attenuated; the residual vibration amplitude of the payload platform is only $24.5 \%$ of the open loop. Compared with the base platform, the average vibration attenuation rate is $92.3 \%$ in this condition. Further, Figure 20 and Table 3 show that the vibration amplitude in the time domain is only about $\pm 8 \mu \mathrm{m} / \mathrm{s}$ in the closed loop (AHC: feedback and feedforward) state. The amplitude of vibration can be further attenuated in the time domain; the remaining vibration of the payload platform is only $16.3 \%$. Compared with the base platform, the average vibration attenuation rate is $94.8 \%$.

5.3. Discussions. From the results, it can be seen that the various DOFs mode decoupling of the vibration isolation system is basically achieved with the assistance of the accurate dynamic model. Experimental results from the frequency domain show that the high frequency of the passive state is greatly attenuated. Nevertheless, there is a sharp resonance peak amplitude when the system is in the open loop state. It means that the vibration signal will not be attenuated but is greatly enlarged. Closed loop control with sky-hook damping feedback is employed for solving the problem of the open state. The amplitude of the resonant peak is decreased by $21.37 \mathrm{~dB} / 23.06 \mathrm{~dB} / 20.83 \mathrm{~dB}$, and simultaneously the high attenuation rate of the high frequency is constant.

In order to further improve the performance and reduce the residual vibration of the payload platform, LLPC feedforward control is adopted. Compared with the open loop, the amplitude of the resonance peak is decreased by $24.62 \mathrm{~dB} / 29.17 \mathrm{~dB} / 23.58 \mathrm{~dB}$. And the active effective bandwidth is extended to the midfrequency region. Simultaneously AHC can ensure the high performance of additional frequency range. Experimental results from the time domain show that the remaining vibration of payload platform in the AHC state is only $16.3 \%$ of that in the passive state. The AHC improves vibration suppression on base platform and further verifies the effectiveness of the proposed hybrid algorithm.

\section{Concluding Remarks}

To describe the dynamic characteristic of the six DOFs AVIS applied in the ultra-precision field more clearly, a coordinate vector conversion from unit level to system level is mentioned firstly according to the layout of the ultra-precision AVIS. And simultaneously, a system level dynamic model is constructed with the help of the transformed coordinate vector. Further, in order to obtain a wider effective frequency band and a higher performance of vibration isolation, an AHC technology is introduced in this paper. AHC technology comprises a FFAC technology and a FBAC technology. Absolute velocity feedback is employed to establish a skyhook damping technology in FBAC technology. Velocity feedforward of base platform is adopted to build a LLPC control technology in FFAC technology. Based on the establishment of the dynamic model and the research of active control, an experimental platform of six DOFs active AVIS which consists of three isolators is set up in super-clean lab.

The experimental results of the spectrum testing and analysis system show that the amplitude of the resonant peak is reduced to $-8.95 \mathrm{~dB} /-8.38 \mathrm{~dB} /-3.60 \mathrm{~dB}$ in $X / Y / Z$ directions. Compared with the open loop, the amplitude of the resonance peak is decreased by $24.62 \mathrm{~dB} /$ $29.17 \mathrm{~dB} / 23.58 \mathrm{~dB}$. The amplitude in $10 \mathrm{~Hz}$ is reduced to $-47.58 \mathrm{~dB} /-44.64 \mathrm{~dB} /-41.14 \mathrm{~dB}$, respectively; the vibration attenuation rate is $99.58 \% / 99.41 \% / 99.12 \%$. And the active effective bandwidth is extended to the midfrequency region of $0.25 \mathrm{~Hz} \sim 32.82 \mathrm{~Hz} / 0.41 \mathrm{~Hz} \sim 45.74 \mathrm{~Hz} / 0.23 \mathrm{~Hz} \sim 14.38 \mathrm{~Hz}$. The vibration amplitude in the time domain is only about $\pm 8 \mu \mathrm{m} / \mathrm{s}$ in AHC state. The amplitude of vibration can be further attenuated in the time domain; the remaining vibration of the payload platform is only $16.3 \%$. Based on the conclusions above, the AHC algorithm which comprises a sky-hook damping algorithm and a LLPC control algorithm is verified to be effective on vibration isolation, respectively, from the frequency domain and time domain.

\section{Competing Interests}

The authors declare that there is no conflict of interests regarding the publication of this paper.

\section{Acknowledgments}

The work was supported by the National Natural Science Foundation of China (no. 51435006, no. 51421062).

\section{References}

[1] A. Preumont, M. Horodinca, I. Romanescu et al., "A six-axis single-stage active vibration isolator based on Stewart platform," Journal of Sound and Vibration, vol. 300, no. 3-5, pp. 644-661, 2007. 
[2] A. M. Abakumov and G. N. Miatov, "Control algorithms for active vibration isolation systems subject to random disturbances," Journal of Sound and Vibration, vol. 289, no. 4-5, pp. 889-907, 2006.

[3] B. N. Agrawal and H.-J. Chen, "Algorithms for active vibration isolation on spacecraft using a Stewart platform," Smart Materials \& Structures, vol. 13, no. 4, pp. 873-880, 2004.

[4] A. Preumont, A. François, F. Bossens, and A. Abu-Hanieh, "Force feedback versus acceleration feedback in active vibration isolation," Journal of Sound and Vibration, vol. 257, no. 4, pp. 605-613, 2002.

[5] A. Preumont, J.-P. Dufour, and C. Malekian, "Active damping by a local force feedback with piezoelectric actuators," Journal of Guidance, Control, and Dynamics, vol. 15, no. 2, pp. 390-395, 1992.

[6] M. Yasuda, T. Osaka, and M. Ikeda, "Feedforward control of a vibration isolation system for disturbance suppression," in Proceedings of the 35th IEEE Conference on Decision and Control, pp. 1229-1233, Kobe, Japan, December 1996.

[7] F.-Y. Wang, “The exact and unique solution for phase-lead and phase-lag compensation," IEEE Transactions on Education, vol. 46, no. 2, pp. 258-262, 2003.

[8] B. Yan, M. J. Brennan, S. J. Elliott, and N. S. Ferguson, "Active vibration isolation of a system with a distributed parameter isolator using absolute velocity feedback control," Journal of Sound and Vibration, vol. 329, no. 10, pp. 1601-1614, 2010.

[9] T. H. Yan, H. Y. Pu, X. D. Chen, Q. Li, and C. Xu, "Integrated hybrid vibration isolator with feedforward compensation for fast high-precision positioning X/Y tables," Measurement Science and Technology, vol. 21, no. 6, Article ID 065901, 2010.

[10] K. S. Yeung, K. W. Wong, and K.-L. Chen, "A non-trial-anderror method for lag-lead compensator design," IEEE Transactions on Education, vol. 41, no. 1, pp. 76-80, 1998.

[11] R. Pena-Alzola, M. Liserre, F. Blaabjerg, R. Sebastian, J. Dannehl, and F. W. Fuchs, "Systematic design of the lead-lag network method for active damping in lcl-filter based three phase converterss," IEEE Transactions on Industrial Informatics, vol. 10, no. 1, pp. 43-52, 2014.

[12] D. Thayer, M. Campbell, J. Vagners, and A. Von Flotow, "Sixaxis vibration isolation system using soft actuators and multiple sensors," Journal of Spacecraft and Rockets, vol. 39, no. 2, pp. 206-212, 2002.

[13] W. Wu, X. Chen, and Y. Shan, "Analysis and experiment of a vibration isolator using a novel magnetic spring with negative stiffness," Journal of Sound and Vibration, vol. 333, no. 13, pp. 2958-2970, 2014.

[14] C. R. Fuller and A. H. von Flotow, "Active control of sound and vibration," IEEE Control Systems, vol. 15, no. 6, pp. 9-19, 1995.

[15] J. Fu, P. Li, Y. Wang, G. Liao, and M. Yu, "Model-free fuzzy control of a magnetorheological elastomer vibration isolation system: analysis and experimental evaluation," Smart Materials and Structures, vol. 25, no. 3, Article ID 035030, 2016.

[16] W. S. Robertson, M. R. F. Kidner, B. S. Cazzolato, and A. C. Zander, "Theoretical design parameters for a quasi-zero stiffness magnetic spring for vibration isolation," Journal of Sound and Vibration, vol. 326, no. 1-2, pp. 88-103, 2009.

[17] A. Valeev, A. Zotov, and S. Kharisov, "Designing of compact low frequency vibration isolator with quasi-zero-stiffness," Journal of Low Frequency Noise, Vibration and Active Control, vol. 34, no. 4, pp. 459-474, 2015.
[18] H. Pu, X. Chen, Z. Zhou, and X. Luo, "Six-degree-of-freedom active vibration isolation system with decoupled collocated control," Proceedings of the Institution of Mechanical Engineers, Part B: Journal of Engineering Manufacture, vol. 226, no. 2, pp. 313-325, 2012.

[19] Q.-G. Wang, Z. Ye, and C. C. Hang, "Tuning of phase-lead compensators for exact gain and phase margins," Automatica, vol. 42, no. 2, pp. 349-352, 2006. 


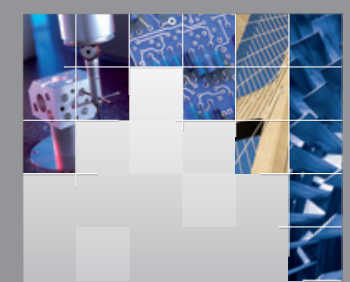

\section{Enfincering}
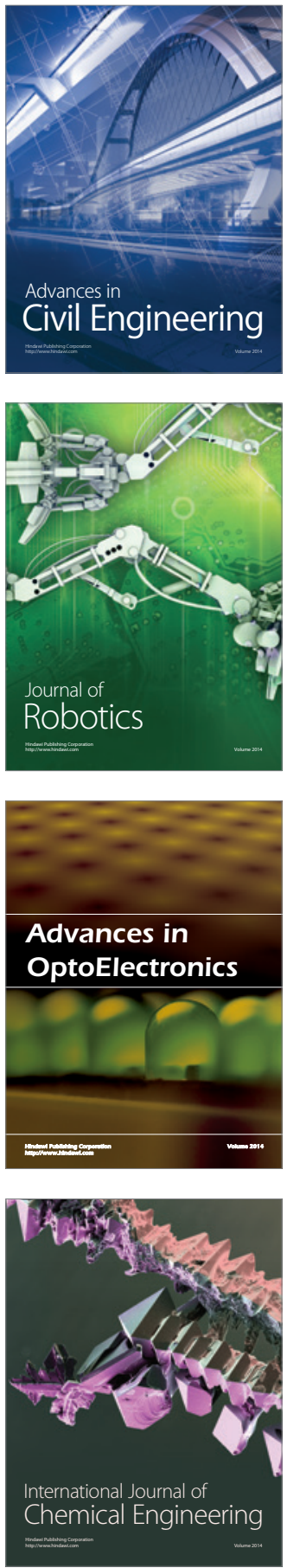

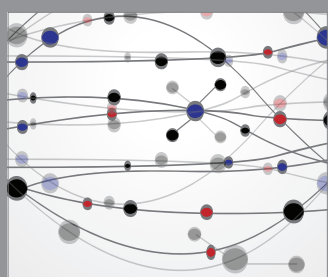

The Scientific World Journal

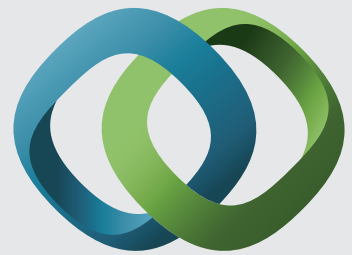

\section{Hindawi}

Submit your manuscripts at

https://www.hindawi.com
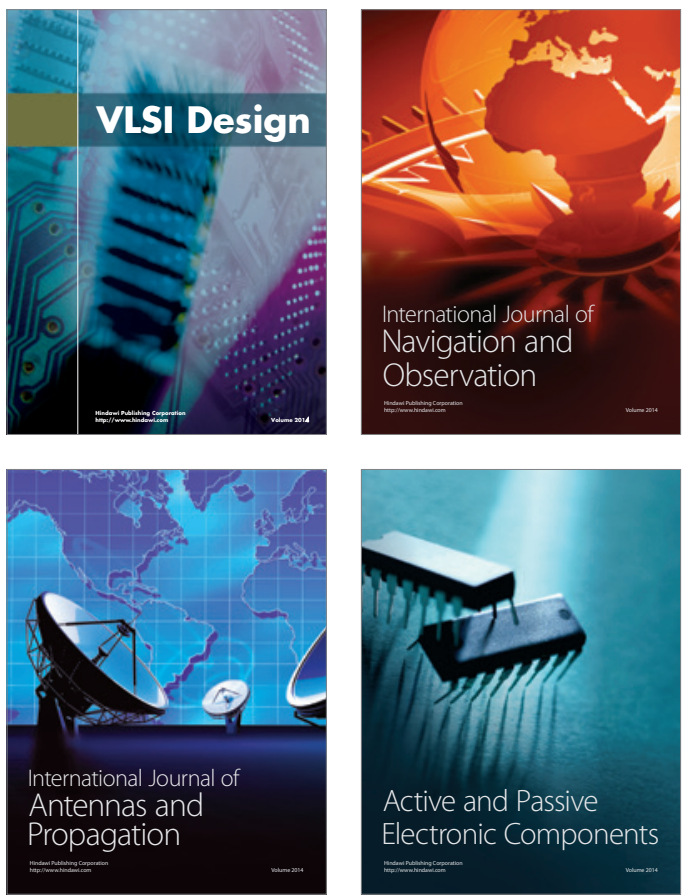
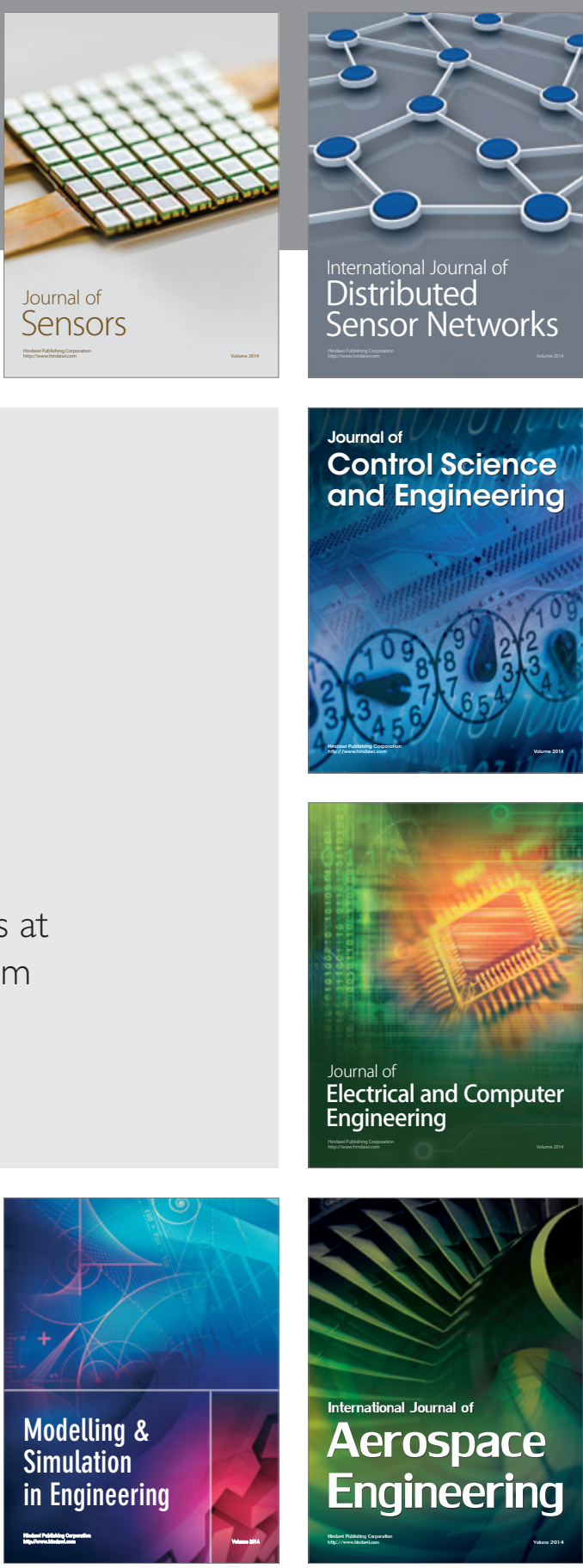

International Journal of

Distributed

Sensor Networks

$-$

Joumal of

Control Science

and Engineering
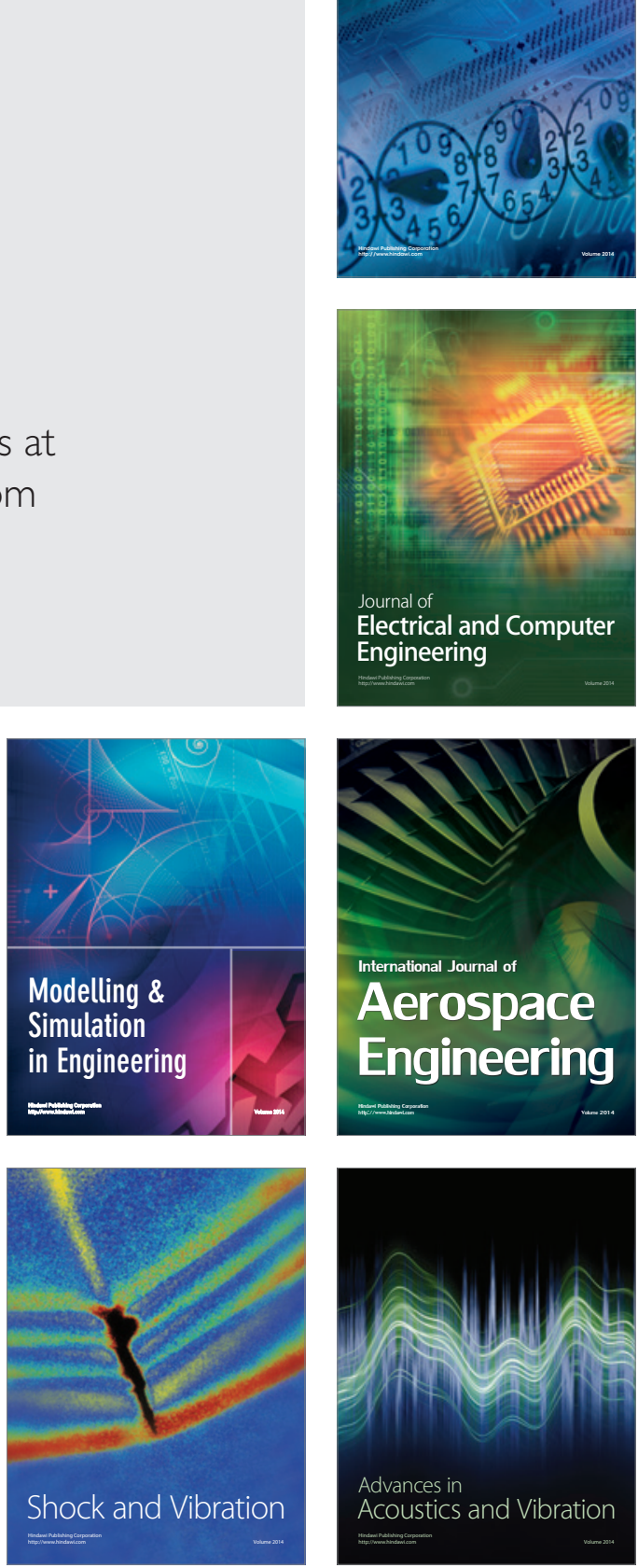